\title{
Comparative analysis of diversity and utilization of edible plants in arid and semi-arid areas in Benin
}

\author{
Alcade C Segnon* and Enoch G Achigan-Dako
}

\begin{abstract}
Background: Agrobiodiversity is said to contribute to the sustainability of agricultural systems and food security. However, how this is achieved especially in smallholder farming systems in arid and semi-arid areas is rarely documented. In this study, we explored two contrasting regions in Benin to investigate how agroecological and socioeconomic contexts shape the diversity and utilization of edible plants in these regions.

Methods: Data were collected through focus group discussions in 12 villages with four in Bassila (semi-arid Sudano-Guinean region) and eight in Boukoumbé (arid Sudanian region). Semi-structured interviews were carried out with 180 farmers (90 in each region). Species richness and Shannon-Wiener diversity index were estimated based on presence-absence data obtained from the focus group discussions using species accumulation curves.

Results: Our results indicated that 115 species belonging to 48 families and 92 genera were used to address food security. Overall, wild species represent $61 \%$ of edible plants collected $(60 \%$ in the semi-arid area and $54 \%$ in the arid area). About 25\% of wild edible plants were under domestication. Edible species richness and diversity in the semi-arid area were significantly higher than in the arid area. However, farmers in the arid area have developed advanced resource-conserving practices compared to their counterparts in the semi-arid area where slash-and-burn cultivation is still ongoing, resulting in natural resources degradation and loss of biodiversity. There is no significant difference between the two areas for cultivated species richness. The interplay of socio-cultural attributes and agroecological conditions explains the diversity of food plants selected by communities.

Conclusions: We conclude that if food security has to be addressed, the production and consumption policies must be re-oriented toward the recognition of the place of wild edible plants. For this to happen we suggest a number of policy and strategic decisions as well as research and development actions such as a thorough documentation of wild edible plants and their contribution to household diet, promotion of the "bringing into cultivation" practices, strengthening of livestock-crop integration.
\end{abstract}

Keywords: Benin, Dry areas, Edible food plants, Food security, Species richness, Socio-cultural attributes, Farming practices

\footnotetext{
* Correspondence: alcadese@gmail.com

Horticulture and Genetics Unit, Faculty of Agronomic Sciences, University of Abomey-Calavi, 01 BP 526, Cotonou, Republic of Benin
} 


\section{Background}

Agrobiodiversity is understood as "the variety and variability of living organisms that contribute to food and agriculture in the broadest sense, and that are associated with cultivating crops and rearing animals within ecological complexes" [1,2]. A sustainable utilization of agrobiodiversity and associated ecosystem services through diversified farming systems is advocated to be a robust approach for addressing food security and the sustainability of agricultural systems [3-8]. However, how agrobiodiversity is used to address food security and sustainability of agricultural systems in smallholder farming systems in arid and semiarid areas of West Africa needs to be investigated and documented so as to inform policy decisions. In fact, the challenge of providing sufficient food for the increasing population while preserving natural resources is higher in arid and semi-arid areas $[9,10]$. In these areas, agricultural production systems are faced not only with persistent water scarcity and frequent drought, but also with high climatic variability, land degradation, desertification, and widespread poverty [9]. The strong climatic variations and irregular rainfalls that characterized agro-ecosystems in dry areas make harvest of staple and cash crops highly uncertain, especially in West African Sahel and dry savannas [11]. These constraints are expected to intensify as a result of population growth, urbanization and climate change, which will likely exacerbate food insecurity in these areas, that are already vulnerable to hunger and under-nutrition [12]. In this context, increased knowledge of the functionalities of agrobiodiversity will help build the social and natural science evidence-base to allow formulation of adequate intensification strategies [13]. These context- and location-specific strategies require a clear understanding of food production and consumption systems.

Previous investigations in West Africa explored the relationships that communities have developed with their environment and surrounding biological resources, including the utilization of plant resources. From those previous findings, we understood that the value and utilization of plant resources in communities are influenced by sociolinguistic membership [14-22] and to some extent geographical contexts $[23,24]$. However, gap still exists in the understanding of how ecological and socioeconomic contexts shape the utilization of agrobiodiversity and its contribution to food security in this region. Most studies focus on the consumption and variation of knowledge of single species (e.g. Parkia biglobosa [14], Sclerocarya birrea [15], Tamarindus indica [22,25], Blighia sapida [16], Adansonia digitata [26]) or categories of species (e.g. woody species $[27,28]$, Non-Timber Forest Products species $[20,29]$, vegetables species $[24,30])$ and the linkage between agrobiodiversity, food consumption and security was partially addressed. Food security is a complex condition with four key dimensions namely food availability, food utilization, food accessibility and food system stability [31]. Understanding how food security is achieved in arid and semi arid areas while sustaining the use of agrobiodiversity will certainly provide insight into plant resources preservations mechanisms, food production strategies, and sustainable livelihoods.

The objectives of this study are to assess the diversity and utilization of edible plant resources, and analyze farming practices in relation to agroecological contexts in agricultural communities of two contrasting regions in Benin. The following questions were addressed in this paper: (1) What are the edible plants used in the arid and the semi-arid regions of Benin and what is the place of wild resources? (2) Is the diversity of edible plants used in the semi-arid and arid regions the same? (3) Are ecological differences between the arid versus semi-arid regions reflected in differences in farming practices and use of edible plant resources? (4) Are edible plants' choice and utilization similar in communities of the arid and the semi-arid regions? We hypothesized that the use of edible plant resources (wild and cultivated) is affected by agroecological conditions, sociolinguistic attributes and farming practices of rural communities.

\section{Methods}

\section{Study area}

This study was conducted in two ecological regions of Benin (West Africa) namely the Sudanian and the Sudano-Guinean regions. Benin is located on the Atlantic coast, and borders Nigeria to the east, Togo to the west, and Burkina Faso to the northwest, and Niger to the north (Figure 1). The vegetation pattern shows a humidity gradient northward as a result of the joint effects of the climate and the soils [32].

The Sudanian region is a woodland and savanna region with ferruginous soils. The rainfall is unimodal with a mean annual for about $1000 \mathrm{~mm}$ (Table 1). The temperature ranges from 24 to $31^{\circ} \mathrm{C}[32,33]$. The main sociolinguistic groups are Bariba, Fulani and Otamari and related sociolinguistic groups [34]. Farming systems are mainly based on cotton and cereal cultivation and livestock breeding. In the Western part, farming systems are limited by both land degradation and availability leading to population migration into central part of the country [35].

The Sudano-Guinean region is a transitional zone between the Guinean forests in the south and the Sudanian woodlands and savannas in the north and is characterized by a vegetation mosaic of forest islands, gallery forests, and savannas. The rainfall is unimodal and lasts for about 200 days with an annual mean rainfall varying from 1100 to $1300 \mathrm{~mm}$ (Table 1). The temperature varies from 25 to $29^{\circ} \mathrm{C}[32,33]$. The main sociolinguistic groups are Fon, Yoruba-Nagot and related sociolinguistic groups [34]. 


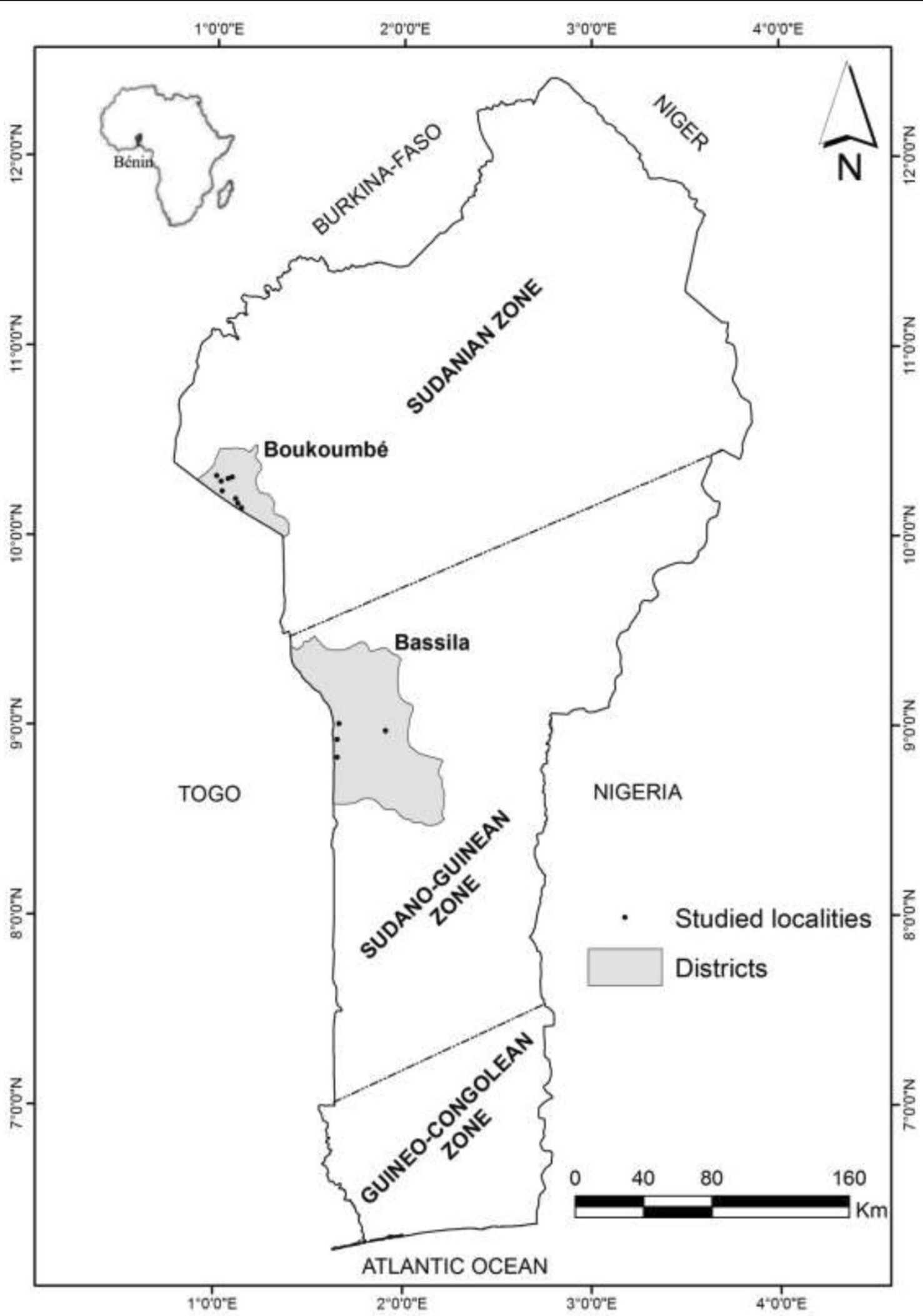

Figure 1 Location of the study areas. 
Table 1 Bio-geographical and socioeconomic characteristics of the study areas

\begin{tabular}{|c|c|c|}
\hline & Semi-arid zone (Bassila) & Arid zone (Boukoumbé) \\
\hline Biophysical gradient & Sudano-Guinean $\left(7^{\circ} 30-9^{\circ} 30 \mathrm{~N}\right)$ region & Sudanian region $\left(9^{\circ} 30-12^{\circ} \mathrm{N}\right)$ region \\
\hline Annual rainfall & $1100-1300 \mathrm{~mm}$ & $900-1100 \mathrm{~mm}$ \\
\hline Active vegetation period & 200 days & 145 days \\
\hline \multicolumn{3}{|l|}{ Relative Humidity } \\
\hline Min & $30-70 \%$ & $<30 \%$ \\
\hline Max & $<80 \%$ & $45-75 \%$ \\
\hline Temperature & 25 to $29^{\circ} \mathrm{C}$ & 24 to $31^{\circ} \mathrm{C}$ \\
\hline Forest cover & $\begin{array}{l}50 \% \text { of the total area of the municipality of Bassila is covered } \\
\text { by forest reserves [Monts Kouffè Forest Reserve ( } 201000 \text { ha), } \\
\text { Pénéssoulou Forest Reserve ( } 5470 \text { ha), Bassila Forest Reserve } \\
\text { ( } 3320 \text { ha) and Wari Maro Forest Reserve ( } 1 / 3 \text { of } 107500 \text { ha)] }\end{array}$ & Not available \\
\hline Farming systems & Cereal- (maize and sorghum) and Yam-based & Cereal-based (sorghum and pearl millet) \\
\hline \multicolumn{3}{|l|}{ Socioeconomic gradient } \\
\hline Food insecurity & Moderate food insecurity & Severe food insecurity \\
\hline \multirow[t]{3}{*}{ Poverty incidence } & Low & High \\
\hline & Less than $40 \%$ of poor household & $72 \%$ of poor household \\
\hline & $20 \%$ of population living in extreme poverty & $51 \%$ of population living in extreme poverty \\
\hline $\begin{array}{l}\text { Number of villages surveyed in each } \\
\text { municipality }\end{array}$ & 04 & 08 \\
\hline $\begin{array}{l}\text { Sociolinguistic groups surveyed } \\
\text { (number of villages) in each municipality }\end{array}$ & Ditamari (01), M’Bermé (01), Nagot (01), Lokpa (01) & Ditamari (06), M’Bermé (02) \\
\hline Number of respondents & 90 & 90 \\
\hline
\end{tabular}

Data assembled from Adomou [32], Akoègninou et al. [33], Bongi et al. [38], MAEP [39].

Other ethnic groups found include Otamari, Yom-Lokpa and related sociolinguistic ethnics groups. These ethnic groups represent two sociolinguistic groups that form the principal actors of migratory dynamics in Benin [23,36]. Members of these groups are motivated to leave their homes in the hilly and over-populated North-West part to the central part in search of the virgin and fertile lands [23,35-37]. Fulani herders are also found in the SudanoGuinean region because of their nomadic pastoralist lifestyle. The yam-based cropping systems are dominant [35] while rice cultivation is also important. Cotton and cashew nut are the major cash crops in the Sudano-Guinean zone.

Based on biophysical and socio-economic gradients $[32,33,38,39]$, we selected Boukoumbé and Bassila municipalities for field investigations (Table 1, Figure 1). Boukoumbé $\left(10^{\circ} 10^{\prime} 36.1^{\prime \prime} \mathrm{N}\right.$ and $\left.01^{\circ} 06^{\prime} 22.0^{\prime \prime} \mathrm{E}\right)$ is located in Atacora department (north-western Benin) and belongs to the arid Sudanian region. Main ethnic groups in Boukoumbé include Ditamari, M'Berme, Natimba and Berba, which form the Otamari socilolinguistic group [34]. Otammari ethnic groups compose $92.4 \%$ of the population of Boukoumbé [34]. Traditional social system of the Otammari is based on crop production [36,40]. Limited access to arable land due to the Atakora mountain chain and land degradation lead Otammari people to leave their homes to the central part of the country [23,35-37]. The total population of Boukoumbé is 60568 with a population density of 58 habitants per $\mathrm{km}^{2}$ [41]. The total land area is $1036 \mathrm{~km}^{2}$. Eighty six percent of household depend primarily on agriculture for their livelihood [41]. About $72 \%$ of households in the department of Atacora (in which belongs Boukoumbé) are poor (the highest proportion at national level) and $51 \%$ of its population live in extreme poverty [38]. To reduce this severe food insecurity an emergency programme of the Benin government was implemented from 2009 to 2011 through various emergency projects [39]. Bassila $\left(09^{\circ} 01^{\prime} 00.1^{\prime \prime} \mathrm{N}\right.$ and $\left.01^{\circ} 40^{\prime} 00.1^{\prime \prime} \mathrm{E}\right)$ is located in Donga department of (upper central Benin) and belongs to the semi-arid Sudano-Guinean region. Main ethnic groups in Bassila include in order of importance Nagot, Anii and Kotokoli ethnic groups [34]. Nagot people are considered as native dwellers of Bassila although they have known to be originated from Yoruba people of Nigeria with whom they share indeed many similarities [23]. Anii and Kotokoli originated from Togo. Other ethnic groups such Otamari, Lokpa and Fulani are found in Bassila due to population migration. Indeed, Bassila as well as other municipalities in the transitional SudanoGuinean zone between the Sudanian zone in the north and the Guinean zone in the south, is a receptacle of strong dynamic migration [23,36]. Otamari are from the Atakora mountain chain region while Lokpa people are 
from municipality of Ouaké (in northern Bassila) [34]. Apart Fulani who has a nomadic pastoralist lifestyle, other ethnic groups in Bassila are mainly tillers [34,40]. The total population of Bassila is 71511 with a population density of 13 habitants per $\mathrm{km}^{2}$ [41]. The total land area is $5661 \mathrm{~km}^{2}$. Currently, Bassila is the second largest municipality of Benin and about half of its total land area is covered with forests. Eighty three percent of household depend primarily on agriculture for their livelihood [41]. About 40\% of households in the department of Donga (in which belongs Bassila) are poor with $20 \%$ of its population living in extreme poverty [38]. Also, Bassila is in a moderate but steady food insecurity situation [39].

\section{Data collection}

In each municipality, we identified main agricultural production zones with local extension service agents. Afterwards, villages were randomly selected in these zones and the number of villages by municipality is proportional (11 to $12 \%$ ) to the total number of villages that each municipality holds. Bassila holds 31 villages whereas Boukoumbé holds 71 villages [41]. We surveyed four villages in Bassila (Adjiro, Aoro-Lokpa, Camp pionier and Mondogui) and eight villages in Boukoumbé (Dimatema, Dipokor 1, Ditchendia, Koukongou, Kounadogou, Koutchata, Okouaro and Tassayota). Each surveyed village exhibit a dominant sociolinguistic group. A sociolinguistic group is understood here as a group in which a member inherits a common language of communication and shares social attributes such as customs, history, and food habits as recognized by Achigan-Dako et al. [24]. We carried out a focus group discussion in each village with about 20 community members and with a balanced representation of men, women, and different age groups. The socially defined age classes (youth - an unmarried individual; an adult - an individual married, but not considered an elder; and elder [42]) were considered. Participants in focus group discussions are community's members locally recognized as knowledgeable about edible plants. Those participants were invited by the chief of the village and their peers. We obtained a permission of the chief of each village before conducting a focus group discussion, and followed the ethical guidelines of the International Society of Ethnobiology [43]. Participants were asked to build a free and an agreed list of edible plants consumed in the villages and to indicate their status (e.g. cultivated, wild or under domestication), utilizations and plant parts used. For local people, a "cultivated" species is a crop plant that is only known to be cultivated in the village; a "wild" species referred to any other food plants ranging from truly wild (entirely wild and collected only when needed) to wild-protected (maintained and protected or preserved in fields and fallows or around habitats with a sort of ownership), and semi-domesticated plants (cultivated in home gardens or in selected parts of cultivated fields where farmers tend to conduct diverse experiments); an "under domestication" species referred to plants of "wild" category that are not considered fully cultivated yet, and are no longer considered truly wild. It includes wildprotected and semi-domesticated plants, and species that are cited as cultivated by some participants and as wild by other during focus group discussion. Cultivated plants that grow spontaneously in the bush or fallow were not considered as wild species. At the end of each focus group, a guided tour was organized in the village to collect vouchers of edible plants previously listed (by their local names) by participants. The vouchers were selected with the help of two informants who were consensually selected among the participants. Species were taxonomically identified following Akoègninou et al. [33]. We also used the illustrated reference book of traditional vegetable species in Benin [44] and an illustrated reference book of trees, shrubs and lianas of dry zones of West Africa [45] to identify plant species. Voucher specimens were sent to the National Herbarium of Benin at University of AbomeyCalavi for further taxonomic confirmation.

In addition to focus group discussions, we carried out semi-structured individual interviews with 90 farmers in each municipality using a questionnaire. Participants were randomly selected and included in the survey after obtaining their verbal prior informed consent. Participant included is an individual who has his farm and hold the decision-making of activities to be implemented on it. Farmers were asked to describe their farming practices starting from land clearing and preparation until post-harvest practices on field.

\section{Data analysis}

To estimate edible plant species richness in the two municipalities, we translated the utilization patterns of village communities into presence-absence data to generate species accumulation curve using EstimateS statistical device [46]. The species accumulation curve represents the number of edible plant species as a function of some measure of the sampling effort employed in the surveys. It yields the asymptotic richness of the assemblage and the computation of the asymmetrical confidence interval of the estimates [46,47]. To assess the diversity of edible plant species in the two municipalities, we used the ShannonWiener index computed in EstimateS based on the species accumulation curve [46].

To compare the estimated edible plants species' richness and diversity between the two areas and between cultivated and wild groups, we performed a $t$-test, a Welch's test or a two-sample Wilcoxon test when appropriate. We used $t$-test when normality and homoscedasticity assumptions were met, Welch's test when normality assumptions was met but not homoscedasticity and two-sample Wilcoxon test when normality and homoscedasticity assumptions 
were not met [48]. Shapiro-Wilk's test for normality and Levene's test for equality of error variances were used to test the normality and the homoscedasticity assumptions respectively. We also used a range of descriptive statistics to account for species diversity and use patterns.

To test if farming practices used by farmers were independent of their agroecological region, we used Fischer's exact test or chi-square test when appropriate. Fischer's exact test was used when some cells of the contingency table had frequencies less than five and these cells represent more than $20 \%$ of the total number of cell in the contingency table $[48,49]$.

To assess relationships among village communities in term of the utilization of edible plants species, we performed a hierarchical cluster analysis based solely on cultivated species, on wild species and then wild and cultivated together. Dendrograms were obtained using the complete linkage algorithm based on Jaccard coefficient of similarity [50] generated from the presence-absence data matrix in which villages were considered as sampling units and species as variables and scored, for each village, as 1 when present or 0 if not. All statistical analyses were performed using R version 3.0.2 [51].

\section{Results}

Diversity and richness of edible plants species and farming context in semi-arid and arid regions

The total edible plant species richness was estimated to vary between 110 and 122 with an absolute value of 115 species (Figure 2a). Species belong to 48 families and 92 genera. Families with the highest genera and species richness include Asteraceae, Poaceae, Anacardiaceae, and Cucurbitaceae. The first two had 6 genera, each one with 8 and 7 species respectively, whereas the last two had 5 genera each one with 7 and 6 species respectively (Table 2). About $48 \%$ of plant families had only one species while
$54 \%$ of them had only one genus. A list of species, with their utilization and part consumed is presented in Table 3. About $50 \%$ of edible plants (57 species) have been cited in more than $50 \%$ of surveyed villages (see Table 3).

Estimation of cultivated species richness indicated 45 species with Confidence Interval (CI) of 42 to 48 species belonging to 23 families and 38 genera. They are dominated by vegetable (47\%) species (e.g. Abelmoschus esculentus, Corchorus olitorius, Solanum macrocarpon, Vernonia amygdalina) and fruit (20\%) species (e.g. Citrus sinensis, Mangifera indica, Psidium guajava). Cereals (e.g. Oryza sativa, Sorghum bicolor, Zea mays) and pulses (e.g. Arachis hypogea, Glycine max, Vigna unguiculata) accounted for $24 \%$ of cultivated species and roots and tubers for $13 \%$ (e.g. Colocasia esculenta, Dioscorea cayenensis-rotundata complex, Ipomoea batatas, Manihot esculenta).

The estimated wild edible species richness indicated 70 species (CI: 66-76) representing $61 \%$ of edible plants collected. They also represent $46 \%$ of the most cited species (species cited in more than $50 \%$ of surveyed villages) by communities (Table 3). About $25 \%$ of them are under domestication and had dual status depending on the village. Some species are entirely wild and collected only when needed (e.g., Ceratotheca sesamoides, Cissus populnea), others are maintained in agricultural environments (fields and fallow) or around habitats (e.g., Adansonia digitata, Vitellaria paradoxa, Parkia biglobosa, Blighia sapida) and others have dual status depending on the village (e.g., Sesamum radiatum, Hibiscus asper, Justicia tenella, Corchorus tridens, Talinum triangulare). Wild edible species belong to 38 families and 61 genera. Vegetable species represent about $57 \%$ while fruit species account for roughly $47 \%$ of wild edible plants collected. Several life forms were recorded, but dominated by trees (36\%) and herbaceous species (33\%). Many wild species (41\%) have
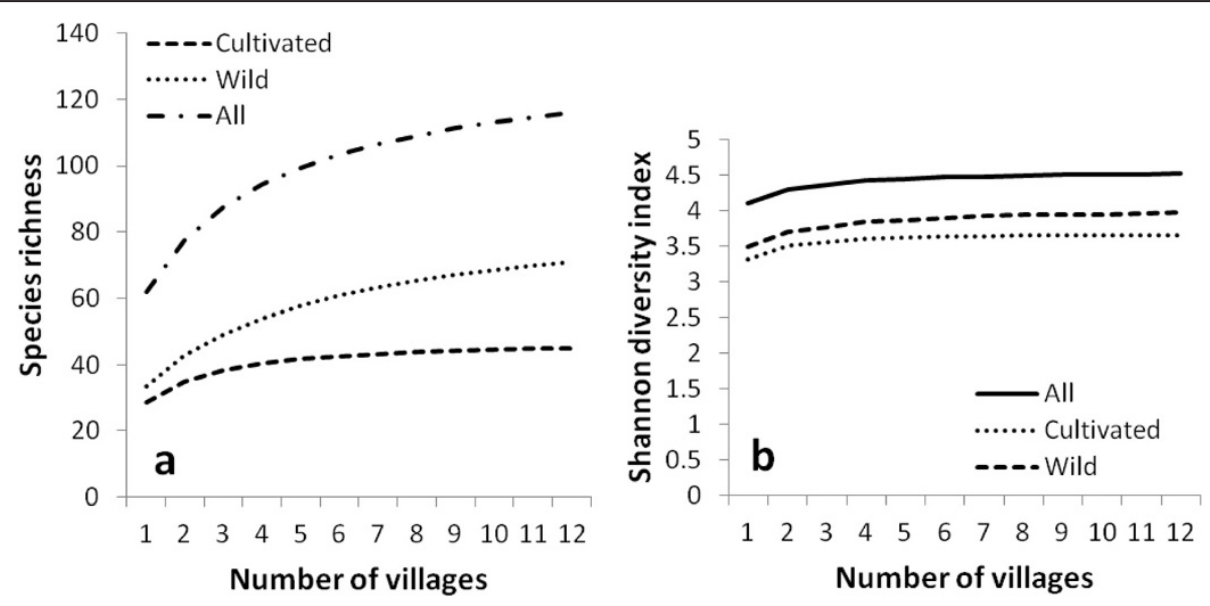

Figure 2 Estimated species richness (a) and Shannon diversity (b) for the edible food plant species in two arid (Boukoumbé) and semi-arid (Bassila) areas of Benin based on incidence data. 
Table 2 Species and genera richness and relative frequency of edible plant families

\begin{tabular}{|c|c|c|c|}
\hline Family & $\begin{array}{l}\text { Genera } \\
\text { richness }\end{array}$ & $\begin{array}{l}\text { Species } \\
\text { richness }\end{array}$ & $\begin{array}{l}\text { Frequency } \\
\text { (\%) }\end{array}$ \\
\hline Asteraceae & 6 & 8 & 0.07 \\
\hline Poaceae & 6 & 7 & 0.06 \\
\hline Anacardiaceae & 5 & 7 & 0.06 \\
\hline Cucurbitaceae & 5 & 6 & 0.05 \\
\hline Leg-Caesalpinioideae & 4 & 5 & 0.04 \\
\hline Leg-Papilionoideae & 4 & 5 & 0.04 \\
\hline Arecaceae & 4 & 4 & 0.03 \\
\hline Amaranthaceae & 2 & 4 & 0.03 \\
\hline Solanaceae & 2 & 4 & 0.03 \\
\hline Tiliaceae & 2 & 4 & 0.03 \\
\hline Moraceae & 1 & 4 & 0.03 \\
\hline Bombacaceae & 3 & 3 & 0.02 \\
\hline Euphorbiaceae & 3 & 3 & 0.02 \\
\hline Lamiaceae & 2 & 3 & 0.02 \\
\hline Malvaceae & 2 & 3 & 0.02 \\
\hline Pedaliaceae & 2 & 3 & 0.02 \\
\hline Rutaceae & 2 & 3 & 0.02 \\
\hline Annonaceae & 2 & 2 & 0.01 \\
\hline Araceae & 2 & 2 & 0.01 \\
\hline Myrtaceae & 2 & 2 & 0.01 \\
\hline Rubiaceae & 2 & 2 & 0.01 \\
\hline Sapindaceae & 2 & 2 & 0.01 \\
\hline Vitaceae & 2 & 2 & 0.01 \\
\hline Acanthaceae & 1 & 2 & 0.01 \\
\hline Apocynaceae & 1 & 2 & 0.01 \\
\hline Asclepiadaceae & 1 & 1 & 0.00 \\
\hline Balanitaceae & 1 & 1 & 0.00 \\
\hline Basellaceae & 1 & 1 & 0.00 \\
\hline Boraginaceae & 1 & 1 & 0.00 \\
\hline Bromeliaceae & 1 & 1 & 0.00 \\
\hline Capparaceae & 1 & 1 & 0.00 \\
\hline Caricaceae & 1 & 1 & 0.00 \\
\hline Chrysobalanaceae & 1 & 1 & 0.00 \\
\hline Clusiaceae & 1 & 1 & 0.00 \\
\hline Cochlospermaceae & 1 & 1 & 0.00 \\
\hline Combretaceae & 1 & 1 & 0.00 \\
\hline Convolvulaceae & 1 & 1 & 0.00 \\
\hline Cyperaceae & 1 & 1 & 0.00 \\
\hline Dioscoreaceae & 1 & 1 & 0.00 \\
\hline Ebenaceae & 1 & 1 & 0.00 \\
\hline Leg-Mimosoideae & 1 & 1 & 0.00 \\
\hline Loganiaceae & 1 & 1 & 0.00 \\
\hline Meliaceae & 1 & 1 & 0.00 \\
\hline
\end{tabular}

Table 2 Species and genera richness and relative frequency of edible plant families (Continued)

\begin{tabular}{llll}
\hline Moringaceae & 1 & 1 & 0.00 \\
Musaceae & 1 & 1 & 0.00 \\
Portulacaceae & 1 & 1 & 0.00 \\
Sapotaceae & 1 & 1 & 0.00 \\
Verbenaceae & 1 & 1 & 0.00 \\
\hline
\end{tabular}

multiple edible parts, including edible leaves, fruits, flowers, pulps, kernel, and/or seeds (e.g. Adansonia digitata, Annona senegalensis, Blighia sapida, Cissus populnea). About $96 \%$ of herbs are used as vegetables whereas about $80 \%$ of tree species provide fresh fruits (Table 4). Shrubs provide fruits $(56 \%)$ and also used as vegetable (39\%). Other uses (about 50\%) were recorded for shrubs and included purgative and laxative (e.g. Tamarindus indica), stimulation of milk production in lactating women (e.g. Zanthoxylum zanthoxyloides), toothbrush (e.g. Vernonia amygdalina). Comparison of estimated richness between wild and cultivated species indicated that wild plant richness is significantly higher than cultivated species richness (Figure 2a, $\mathrm{p}<0.001$ ).

The estimation of species richness per region indicated 99 species (CI: $94-104)$ with about $60 \%$ of wild species (59 species) in Bassila and 82 species (CI: 79-89) with about $54 \%$ of wild species (44 species) in Boukoumbé. Overall, edible species richness in Bassila was significantly higher than in Boukombé (Figure 3a, p <0.05). The same trend was observed when taking into account only wild species (Figure $3 \mathrm{~b}, \mathrm{p}<0.01)$. However, there is no significant difference between the two areas when considering only cultivated species (Figure 3c, p = 0.1334). Thirty five out of 45 cultivated species collected are shared by the two areas (e.g. Zea mays, Sorghum bicolour, Vigna unguiculata, Arachis hypogea, D. cayenensis-rotundata complex, Manihot esculenta, Abelmoschus esculentus, Corchorus olitorius, Mangifera indica, Psidium guajava). Main species only cultivated in Bassila include Ananas comosus and Elaeis guineensis. Digitaria exilis, D. iburua and Pennisetum glaucum are the three species only found in Boukoumbé.

The Shannon-Wiener diversity index was high, up to 4.5 for all categories and followed an asymptotic function against the sample frequency (Figure $2 \mathrm{~b}$ ). Wild species diversity is significantly higher than cultivated species diversity (Figure $2 \mathrm{~b}, \mathrm{p}<0.001$ ). The Shannon-Wiener diversity index was equally high in the two regions. It was close to 4.5 and 4.2 in Bassila and Boukoumbé respectively. Overall, edible species diversity in the semi-arid Sudano-Guinean area was significantly higher than in the arid Sudanian area (Figure $4 \mathrm{a}, \mathrm{p}<0.001$ ). The same trend is observed when taking into account only wild species (Figure $4 \mathrm{~b}, \mathrm{p}<0.001$ ) and also cultivated species only (Figure $4 \mathrm{c}, \mathrm{p}<0.001$ ). 
Table 3 Edible plant species collected in two arid (Boukoumbé) and semi-arid (Bassila) areas of Benin

\begin{tabular}{|c|c|c|c|c|c|c|c|c|c|}
\hline $\begin{array}{l}\text { Voucher } \\
\text { specimen } \\
\text { code }\end{array}$ & Local name ${ }^{1}$ & Species & Family & $\begin{array}{l}\text { Life } \\
\text { form }\end{array}$ & $\begin{array}{l}\text { Phytogeographical } \\
\text { zone }^{2}\end{array}$ & Status $^{3}$ & Utilizations & $\begin{array}{l}\text { Parts } \\
\text { consumed }\end{array}$ & $\begin{array}{l}\text { Frequency of } \\
\text { citations (\%) }\end{array}$ \\
\hline AS015 & $\begin{array}{l}\text { Tinoufanti (D), Tinoussanté (M), Maatou } \\
(\mathrm{L}) \text {, lla }(\mathrm{N})\end{array}$ & Abelmoschus esculentus (L.) Moench & Malvaceae & Herb & $S G, S$ & C & Vegetable & $\begin{array}{l}\text { Leaves, } \\
\text { Fruits }\end{array}$ & 100 \\
\hline AS016 & $\begin{array}{l}\text { Moutonmu, Titookanti (D), Kotôlaxa (L), } \\
\text { Otché, Osché (N) }\end{array}$ & Adansonia digitata $\mathrm{L}$. & Bombacaceae & Tree & $S G, S$ & UD & $\begin{array}{l}\text { Vegetable, } \\
\text { Fruit, } \\
\text { Condiment, } \\
\text { Beverage }\end{array}$ & $\begin{array}{l}\text { Leaves, Pulp, } \\
\text { Seed }\end{array}$ & 100 \\
\hline AS017 & $\begin{array}{l}\text { Munamutimu (D), Yarohu, Waloho, } \\
\text { Sonyonma (L), Abo, Arere (N) }\end{array}$ & Annona senegalensis Pers. & Annonaceae & Shrub & SG, S & W & $\begin{array}{l}\text { Vegetable, } \\
\text { Fruit, } \\
\text { Condiment }\end{array}$ & $\begin{array}{l}\text { Flowers, } \\
\text { Young } \\
\text { leaves, Pulp, } \\
\text { Fruits }\end{array}$ & 100 \\
\hline AS018 & $\begin{array}{l}\text { Tikomaanti, Dikomaanti (D), Nonyrèm, } \\
\text { Nèyèrèm (L), Ekpa (N) }\end{array}$ & Arachis hypogea $\mathrm{L}$. & Leg-Papilionoideae & Herb & $S G, S$ & C & $\begin{array}{l}\text { Pulse, } \\
\text { Vegetable oil }\end{array}$ & Seeds & 100 \\
\hline AS019 & Moufodoom (D), Pèèryu (L), Ishin (N) & Blighia sapida K.D.Koenig & Sapindaceae & Tree & SG, S & UD & $\begin{array}{l}\text { Vegetable, } \\
\text { Condiment }\end{array}$ & $\begin{array}{l}\text { Aril, Young } \\
\text { leaves }\end{array}$ & 100 \\
\hline AS020 & Mufô, Tikonfaati (D), Vèèkou (L), Agougou (N) & Bombax costatum Pellegr. \& Vuillet & Bombacaceae & Tree & SG, S & UD & Vegetable & Flowers & 100 \\
\hline AS021 & Mukpiatimu (D), Kparèèyu (L) Agbon (N) & Borassus aethiopum Mart. & Arecaceae & Tree & $S G, S$ & W & $\begin{array}{l}\text { Fruit, } \\
\text { Vegetable }\end{array}$ & $\begin{array}{l}\text { Pulp, } \\
\text { Seedling }\end{array}$ & 100 \\
\hline AS022 & $\begin{array}{l}\text { Mukanbammu (D), Gemburu (L), Ata } \\
\text { sisebe, Ata eiye (N) }\end{array}$ & Capsicum frutenscens $L$. & Solanaceae & Herb & SG, S & C & Vegetable & Fruits & 100 \\
\hline AS023 & Mukomu (D), Tixoxanté (M), Agougou (N) & Ceiba pentandra (L.) Gaertn. & Bombacaceae & Tree & SG, S & W & Vegetable & $\begin{array}{l}\text { Young } \\
\text { leaves }\end{array}$ & 100 \\
\hline ASO24 & $\begin{array}{l}\text { Tiwadooti (D), Tikpainn'tissêdonté (M), } \\
\text { Xonônm (L), Idjabô (N) }\end{array}$ & Ceratotheca sesamoides Endl. & Pedaliaceae & Herb & $S G, S$ & UD & Vegetable & Leaves & 100 \\
\hline AS025 & Muyua yuam (D), Kpôgolo (N) & Cissus populnea Guill. \& Perr. & Vitaceae & Liana & $S G, S$ & W & $\begin{array}{l}\text { Vegetable, } \\
\text { Condiment }\end{array}$ & $\begin{array}{l}\text { Flowers, } \\
\text { Young } \\
\text { leaves }\end{array}$ & 100 \\
\hline AS026 & $\begin{array}{l}\text { Tikowounkofanti, Yèkotenko (D), } \\
\text { Timoukan'té (M), Mangani (L,N) }\end{array}$ & Colocasia esculenta (L.) Schott & Araceae & Herb & $S G, S$ & C & $\begin{array}{l}\text { Vegetable, } \\
\text { Tuber }\end{array}$ & $\begin{array}{l}\text { Leaves, } \\
\text { Corms }\end{array}$ & 100 \\
\hline AS027 & Mumasôkô (D), lyede (N) & Detarium microcarpum Guill. \& Perr. & Leg-Caesalpinioideae & Tree & $S G, S$ & W & Fruit & Pulp & 100 \\
\hline ASO28 & Yanwaa (D), Hê (L), Ishu (N) & $\begin{array}{l}\text { Dioscorea cayenensis-D. rotundata } \\
\text { species complex }\end{array}$ & Dioscoreaceae & Herb & $S G, S$ & C & Tuber & Tubers & 100 \\
\hline AS029 & $\begin{array}{l}\text { Yêpin, Mupiin (D), Gaaya, Gaayu (L), Igi } \\
\text { dudu, Karan (N) }\end{array}$ & $\begin{array}{l}\text { Diospyros mespiliformis Hochst. ex A. } \\
\text { DC. }\end{array}$ & Ebenaceae & Tree & SG, S & W & Fruit & Pulp & 100 \\
\hline AS030 & Soja (D, L, N) & Glycine max (L.) Merr. & Leg-Papilionoideae & Herb & $S G, S$ & C & Pulse & Grain & 100 \\
\hline AS031 & $\begin{array}{l}\text { Tikwouann'ti (D), Tikonn'té (M), } \\
\text { Ankpaman (L), Kpakpala, Amukan (N) }\end{array}$ & Hibiscus sabdariffa L. & Malvaceae & Herb & $S G, S$ & C & $\begin{array}{l}\text { Vegetable, } \\
\text { Beverage }\end{array}$ & $\begin{array}{l}\text { Leaves, } \\
\text { Flowers }\end{array}$ & 100 \\
\hline AS032 & $\begin{array}{l}\text { Timanuonti (D), Tôxômba (L), Oduku, } \\
\text { Adokwin (N) }\end{array}$ & Ipomoea batatas (L.) Lam. & Convolvulaceae & Herb & SG, S & C & Tuber & Tubers & 100 \\
\hline
\end{tabular}


Table 3 Edible plant species collected in two arid (Boukoumbé) and semi-arid (Bassila) areas of Benin (Continued)

\begin{tabular}{|c|c|c|c|c|c|c|c|c|c|}
\hline AS033 & $\begin{array}{l}\text { Yapeetaa, Bubaturi (D), Manguna (L), } \\
\text { Mangoro (N) }\end{array}$ & Mangifera indica L. & Anacardiaceae & Tree & SG, S & C & Fruit & Fruits & 100 \\
\hline AS034 & $\begin{array}{l}\text { Munuan, Muwassadému (D), Dooso (L), } \\
\text { Igba, Ayidan (N) }\end{array}$ & $\begin{array}{l}\text { Parkia biglobosa (Jacq.) R.Br. ex G. } \\
\text { Don }\end{array}$ & Leg-Mimosoideae & Tree & SG, S & W & $\begin{array}{l}\text { Fruit, } \\
\text { Condiment }\end{array}$ & Pulp, Seeds & 100 \\
\hline AS035 & Mukwamkwam (D) & $\begin{array}{l}\text { Sarcocephalus latifolius (Sm.) E.A. } \\
\text { Bruce }\end{array}$ & Rubiaceae & Shrub & $S G, S$ & W & Fruit & Fruits & 100 \\
\hline AS036 & $\begin{array}{l}\text { Tiwadouanti (D), Tissêdôonté (M), } \\
\text { Touhounôm (L), Dossé (N) }\end{array}$ & $\begin{array}{l}\text { Sesamum radiatum Schumach. \& } \\
\text { Thonn. }\end{array}$ & Pedaliaceae & Herb & SG, S & UD & Vegetable & Leaves & 100 \\
\hline AS037 & $\begin{array}{l}\text { Tiyooti (D), M'la (L), Oka baba, Oka } \\
\text { kpikpa (N) }\end{array}$ & Sorghum bicolor (L.) Moench & Poaceae & Herb & SG, S & C & Cereal & Grain & 100 \\
\hline AS038 & Mupin (D), Ajagbon (N) & Tamarindus indica $\mathrm{L}$. & Leg-Caesalpinioideae & Tree & $S G, S$ & W & Fruit, Beverage & Pulp & 100 \\
\hline AS039 & $\begin{array}{l}\text { Tifinhoun'ti (D), Tikoun'téété (M), } \\
\text { Elimkpataxa (L), Aroman (N) }\end{array}$ & Vernonia amygdalina Delile & Asteraceae & Shrub & SG, S & C & Vegetable & Leaves & 100 \\
\hline AS040 & Yakammwà (D), Tura, Tira (L), Ekpa boro (N) & Vigna subterranea (L.) Verdc. & Leg-Papilionoideae & Herb & SG, S & C & Pulse & Grain & 100 \\
\hline AS041 & $\begin{array}{l}\text { Titoun'ti (D), Tnainyéritonn'té (M), } \\
\text { Tchaassé (L), Ewe, Ewa (N) }\end{array}$ & Vigna unguiculata (L.) Walp. & Leg-Papilionoideae & Herb & SG, S & C & Pulse & Grain & 100 \\
\hline AS042 & Mutaamu (D), Tambéré (L), Emin (N) & Vitellaria paradoxa C.F.Gaertn. & Sapotaceae & Tree & SG, S & UD & $\begin{array}{l}\text { Fruit, } \\
\text { Vegetable oil }\end{array}$ & Fruit, Kernel & 100 \\
\hline AS043 & $\begin{array}{l}\text { Tikowounkofanti, Yèkotenko (D), } \\
\text { Timoukan'té (M), Mangani (L,N) }\end{array}$ & Xanthosoma sagittifolium (L.) Schott & Araceae & Herb & SG, S & C & Tuber & Tubers & 100 \\
\hline AS044 & Dimariyo (D), Manzoo (L), Agbado (N) & Zea mays $\mathrm{L}$. & Poaceae & Herb & SG, S & C & Cereal & Grain & 100 \\
\hline AS045 & Tifaanti (D), Tipanoussanté (M), Yôyô (L,N) & Corchorus olitorius L. & Tiliaceae & Herb & SG, S & C & Vegetable & Leaves & 91.67 \\
\hline AS046 & Mukankanwa (D) & Ficus sycomorus L. & Moraceae & Tree & SG, S & W & $\begin{array}{l}\text { Fruit, } \\
\text { Vegetable }\end{array}$ & $\begin{array}{l}\text { Fruits, Young } \\
\text { leaves }\end{array}$ & 91.67 \\
\hline AS047 & Tikansibouoti (D), Tikli (L) & Hibiscus asper Hook.f. & Malvaceae & Herb & SG, S & UD & Vegetable & $\begin{array}{l}\text { Leaves, } \\
\text { Flowers }\end{array}$ & 91.67 \\
\hline AS048 & Yimwa (D), Murii (L), Resi, Iresi (N) & Oryza sativa $\mathrm{L}$. & Poaceae & Herb & SG, S & C & Cereal & Grain & 91.67 \\
\hline AS049 & $\begin{array}{l}\text { Mumatonmu, Timantounn'ti (D), } \\
\text { Tiwatonn'té (M), Sowarya (L), } \\
\text { Akoumanlapka, Ori nla, Osha koro (N) }\end{array}$ & Vitex doniana Sweet & Verbenaceae & Tree & SG, S & W & $\begin{array}{l}\text { Vegetable, } \\
\text { Fruit }\end{array}$ & $\begin{array}{l}\text { Leaves, } \\
\text { Fruits }\end{array}$ & 91.67 \\
\hline AS050 & $\begin{array}{l}\text { Ifanhanyéi (D), Tixanté (M), Koxolanhoun } \\
(\mathrm{L}) \text {, Ountcho }(N)\end{array}$ & Corchorus tridens $\mathrm{L}$. & Tiliaceae & Herb & SG, S & UD & Vegetable & Leaves & 83.33 \\
\hline AS051 & Mukpiatikakadata (D) & Hyphaene thebaica (L.) Mart. & Arecaceae & Tree & SG, S & W & Fruit & Pulp, Kernel & 83.33 \\
\hline AS052 & $\begin{array}{l}\text { Mussan, Issangnan (D), Jaakpeegna (L), } \\
\text { Aku, Asogika, Aso gidoka (N) }\end{array}$ & Lannea microcarpa Engl. \& K.Krause & Anacardiaceae & Tree & SG, S & W & Fruit & Pulp & 83.33 \\
\hline AS053 & Nyakabu (D), Bémeyu (L), lyeye, Eyeye (N) & Spondias mombin L. & Anacardiaceae & Tree & SG, S & W & Fruit & Pulp & 83.33 \\
\hline AS054 & Ditchéfouwounti, Titchéfouwounti (D), & Cleome gynandra L. & Capparaceae & Herb & $S G, S$ & UD & Vegetable & Leaves & 66.67 \\
\hline
\end{tabular}


Table 3 Edible plant species collected in two arid (Boukoumbé) and semi-arid (Bassila) areas of Benin (Continued)

\begin{tabular}{|c|c|c|c|c|c|c|c|c|c|}
\hline AS001 & $\begin{array}{l}\text { Ipoa, Ipordapia, Ipordawan (D), } \\
\text { Ipogninimè, Iporni (M) }\end{array}$ & Digitaria exilis (Kippist) Stapf & Poaceae & Herb & S & C & Cereal & Grain & 66.67 \\
\hline AS002 & Ipoaga (D) & Digitaria iburua Stapf & Poaceae & Herb & S & C & Cereal & Grain & 66.67 \\
\hline AS055 & Munamênii (D) & Gardenia erubescens Stapf \& Hutch. & Rubiaceae & Shrub & SG, S & W & $\begin{array}{l}\text { Fruit, } \\
\text { Condiment }\end{array}$ & $\begin{array}{l}\text { Flowers, } \\
\text { Fruits, } \\
\text { Young } \\
\text { leaves }\end{array}$ & 66.67 \\
\hline AS003 & Yayomaata (D) & Pennisetum glaucum (L.) R.Br. & Poaceae & Herb & S & C & Cereal & Grain & 66.67 \\
\hline AS056 & $\begin{array}{l}\text { Mupotimu (D), Gbaadagnu (L), Goba, } \\
\text { Ewé goba (N) }\end{array}$ & Psidium guajava $\mathrm{L}$. & Myrtaceae & Shrub & SG, S & C & Fruit & Fruits & 66.67 \\
\hline AS057 & Yapeerka (D), Timaati (L), Tomati (N) & Solanum Iycopersicum L. & Solanaceae & Herb & SG, S & C & Vegetable & Fruits & 66.67 \\
\hline AS058 & Muboborimu (D), Pempeeya (L), Gogo (N) & Strychnos spinosa Lam. & Loganiaceae & Shrub & SG, S & W & $\begin{array}{l}\text { Fruit, } \\
\text { Vegetable }\end{array}$ & $\begin{array}{l}\text { Fruits, Young } \\
\text { leaves }\end{array}$ & 66.67 \\
\hline AS059 & Kaju (D), Akadiya (L), Kaju (N) & Anacardium occidentale $\mathrm{L}$. & Anacardiaceae & Tree & SG, S & C & Fruit & Kernel & 58.33 \\
\hline AS060 & Aléfô (D), Bee kumpeeyu (L), Fotètè (N) & Amaranthus cruentus L. & Amaranthaceae & Herb & SG, S & C & Vegetable & Leaves & 50 \\
\hline AS061 & Muporicoetimu (D) & Balanites aegyptiaca (L.) Delile & Balanitaceae & Tree & SG, S & C & Fruit & Fruits & 50 \\
\hline AS062 & $\begin{array}{l}\text { Tinonyawouti (D), Tipékênonté (M), } \\
\text { Nyaayu (L), Tchôkôyôkôtô (N) }\end{array}$ & Celosia argentea $\mathrm{L}$. & Amaranthaceae & Herb & SG, S & C & Vegetable & Leaves & 50 \\
\hline AS063 & $\begin{array}{l}\text { Mutaarmu (D), Agusi, Teneyu (L), Kaka, } \\
\text { Egusi (N) }\end{array}$ & Lagenaria siceraria (Molina) Standl. & Cucurbitaceae & Herb & SG, S & C & Vegetable & Fruits, Seeds & 50 \\
\hline AS064 & Tifôônouwôti (D), Agbédéxatou (L), Kpaki (N) & Manihot esculenta Crantz & Euphorbiaceae & Shrub & SG, S & C & Root & Roots & 50 \\
\hline AS065 & $\begin{array}{l}\text { Tibòdayati, Tibòsèyenti (D), Tignainté (M), } \\
\text { Assôou (L), Alounmamba, Aribala (N) }\end{array}$ & Ocimum gratissimum L. & Lamiaceae & Herb & SG, S & C & Spice & Leaves & 50 \\
\hline AS004 & $\begin{array}{l}\text { Muganyan, Mounannikmon (D), } \\
\text { Ubamingbu (M) }\end{array}$ & Sclerocarya birrea (A.Rich) Hochst. & Anacardiaceae & Tree & S & W & Fruit, Beverage & Fruits, Pulp & 50 \\
\hline AS066 & $\begin{array}{l}\text { Muwaadonmu (D), Saamu, Nareer (L), } \\
\text { Yonmonti, Nyamoti, Ewe ekutu (N) }\end{array}$ & Sesamum indicum $\mathrm{L}$. & Pedaliaceae & Herb & SG, S & C & Vegetable & $\begin{array}{l}\text { Leaves, } \\
\text { Seeds }\end{array}$ & 50 \\
\hline AS067 & $\begin{array}{l}\text { Tikawounfanti (D), Tikann'té (M), Gboma } \\
\text { (L), Gboma, Kpatakpakô (N) }\end{array}$ & Solanum macrocarpon L. & Solanaceae & Herb & SG, S & C & Vegetable & Leaves & 50 \\
\hline AS068 & $\begin{array}{l}\text { Demumuda daniira (D), Akutongnu, } \\
\text { Afotongnu (L), Orombo didu, Orombo } \\
\text { igun }(\mathrm{N})\end{array}$ & Citrus sinensis (L.) Osbeck & Rutaceae & Tree & SG, S & C & Fruit & Fruits & 41.67 \\
\hline AS005 & Mumasôkô (D), Ogbôgbô (N) & Detarium senegalense J.F.Gmel. & Leg-Caesalpinioideae & Tree & S & W & Fruit & Pulp & 41.67 \\
\hline AS069 & Mupénuamu (D) & Ficus asperifolia Miq. & Moraceae & Shrub & SG, S & W & Fruit & Fruits & 41.67 \\
\hline AS070 & $\begin{array}{l}\text { Tinoukounti (D), Tilétoussi (L), } \\
\text { Djagou-djagou (N) }\end{array}$ & Justicia tenella (Nees) T.Anderson & Acanthaceae & Herb & SG, S & UD & Vegetable & $\begin{array}{l}\text { Leaves, } \\
\text { Shoot, Stem }\end{array}$ & 41.67 \\
\hline AS071 & Tikoun'tééti (D), Arikoro (N) & Vernonia colorata (Willd.) Drake & Asteraceae & Shrub & SG, S & W & Vegetable & Leaves & 41.67 \\
\hline AS072 & Tipébouoti (D), Oubouonou, Ibouoni (M) & Acmella oleracea (L.) R.K.Jansen & Asteraceae & Herb & SG, S & UD & Vegetable & Leaves & 33.33 \\
\hline AS073 & Yapeertora (D), Ibekpe, Igi bekpe (N) & Carica papaya L. & Caricaceae & Tree & $S G, S$ & C & Fruit & Fruits & 33.33 \\
\hline
\end{tabular}


Table 3 Edible plant species collected in two arid (Boukoumbé) and semi-arid (Bassila) areas of Benin (Continued)

\begin{tabular}{|c|c|c|c|c|c|c|c|c|c|}
\hline AS083 & Tinacanti (D), Egusi, Itoô (N) & Citrullus mucosospermus Fursa & Cucurbitaceae & Herb & SG & C & Vegetable & Seeds & 33.33 \\
\hline AS084 & Gbolo (N) & $\begin{array}{l}\text { Crassocephalum crepidioides (Benth.) } \\
\text { S.Moore }\end{array}$ & Asteraceae & Herb & SG & UD & Vegetable & Leaves & 33.33 \\
\hline AS085 & Mupomu (D), Be yiya (L), Igi okpe, Okpe (N) & Elaeis guineensis Jacq. & Arecaceae & Tree & SG & C & Vegetable oil & Pulp & 33.33 \\
\hline AS006 & Munapuo (D) & Ficus dicranostyla Mildbr. & Moraceae & Shrub & $S$ & W & $\begin{array}{l}\text { Fruit, } \\
\text { Vegetable }\end{array}$ & $\begin{array}{l}\text { Fruits, Young } \\
\text { leaves }\end{array}$ & 33.33 \\
\hline AS074 & Mupémi (D) & Lannea acida A.Rich. & Anacardiaceae & Tree & SG, S & W & Fruit & Pulp & 33.33 \\
\hline AS086 & Odôdô (N) & $\begin{array}{l}\text { Launaea taraxacifolia (Willd.) Amin ex } \\
\text { C.Jeffrey }\end{array}$ & Asteraceae & Herb & SG & W & Vegetable & Leaves & 33.33 \\
\hline AS075 & $\begin{array}{l}\text { Mounpêkom (D), Kêpiénouakê (M), } \\
\text { Agdêdêxatou, Lôtaxa (L), Ekégnibo (N) }\end{array}$ & Moringa oleifera Lam. & Moringaceae & Shrub & SG, S & C & Vegetable & $\begin{array}{l}\text { Leaves, } \\
\text { Seeds }\end{array}$ & 33.33 \\
\hline AS076 & $\begin{array}{l}\text { Yèkodiyè (D), Kainton'ko (M), } \\
\text { Kouwoundou (L), Tchidifulè, Yèbè, Iman (N) }\end{array}$ & Solanum aethiopicum L. & Solanaceae & Herb & SG, S & C & Vegetable & $\begin{array}{l}\text { Immature } \\
\text { fruits, Young } \\
\text { leaves }\end{array}$ & 33.33 \\
\hline AS077 & $\begin{array}{l}\text { Yêmontouo (D), Kamplékankann'dê (L), } \\
\text { Odondon, Gbure, Gure (N) }\end{array}$ & Talinum triangulare (Jacq.) Willd. & Portulacaceae & Herb & SG & UD & Vegetable & Leaves & 33.33 \\
\hline AS087 & Faso (D), Saada kuriji (L), Yraha, Eruju (N) & Uvaria chamae P.beauv. & Annonaceae & Shrub & SG & W & Fruit & Pulp & 33.33 \\
\hline AS088 & Mubuo (D), Igi ata (N) & $\begin{array}{l}\text { Zanthoxylum zanthoxyloides (Lam.) } \\
\text { Zepern. \& Timber }\end{array}$ & Rutaceae & Shrub & SG & W & $\begin{array}{l}\text { Vegetable, } \\
\text { Spice }\end{array}$ & $\begin{array}{l}\text { Leaves, Root } \\
\text { bark }\end{array}$ & 33.33 \\
\hline AS078 & Yépètum (D), Paltiyu (L), Otili (N) & Cajanus cajan (L.) Millsp. & Leg-Papilionoideae & Shrub & SG, S & C & Pulse & Seeds & 25 \\
\hline AS089 & Tipeti (D), Elegede (N) & Cucurbita maxima Duchesne & Cucurbitaceae & Herb & SG & C & Vegetable & $\begin{array}{l}\text { Fruits, } \\
\text { Leaves }\end{array}$ & 25 \\
\hline AS090 & Anwin, Iwin (N) & Dialium guineense Willd. & Leg-Caesalpinioideae & Tree & SG & W & Fruit & Pulp & 25 \\
\hline AS007 & Mukankanwamimu (D) & Ficus sur Forssk. & Moraceae & Tree & $S$ & W & Fruit & Fruits & 25 \\
\hline AS091 & Moussannoum, Mussantiwamu (D) & Grewia mollis Juss. & Tiliaceae & Shrub & SG & W & $\begin{array}{l}\text { Vegetable, } \\
\text { Condiment }\end{array}$ & $\begin{array}{l}\text { Flowers, } \\
\text { Fruits, Leaves }\end{array}$ & 25 \\
\hline AS008 & Issian (D) & Lannea barteri (Oliv.) Engl. & Anacardiaceae & Tree & $S$ & W & Fruit & Pulp & 25 \\
\hline AS092 & Aminagnu (L), Ogede (N) & Musa sp. & Musaceae & Herb & SG & C & Fruit & Fruits & 25 \\
\hline AS093 & - & $\begin{array}{l}\text { Neocarya macrophylla (Sabine) } \\
\text { Prance }\end{array}$ & Chrysobalanaceae & Shrub & SG & W & Fruit & Kernel, Pulp & 25 \\
\hline AS009 & Mupeketatié (D) & Paullinia pinnata $\mathrm{L}$. & Sapindaceae & Liana & S & W & Fruit & Pulp & 25 \\
\hline AS094 & Kodjonou (L), Ekuso (N) & Pentadesma butyracea Sabine & Clusiaceae & Tree & SG & W & Vegetable oil & Kernel & 25 \\
\hline AS095 & Tètè dudu wèrè (N) & Amaranthus viridis $\mathrm{L}$. & Amaranthaceae & Herb & SG & W & Vegetable & Leaves & 16.67 \\
\hline AS010 & Tawotatchoyan (D) & Antidesma venosum Tul. & Euphorbiaceae & Shrub & $S$ & W & Fruit & Fruits & 16.67 \\
\hline AS096 & Egusi (L), Itoô (N) & Cucumeropsis mannii Naudin & Cucurbitaceae & Herb & SG & C & Vegetable & Seeds & 16.67 \\
\hline AS079 & Yanaacemmora (D), Ofio, Omu, Amu (N) & Cyperus esculentus L. & Cyperaceae & Herb & SG, S & C & Tuber & Tubers & 16.67 \\
\hline AS097 & Suyouxo (L), Ogbé akuko (N) & Heliotropium indicum L. & Boraginaceae & Herb & SG & W & Vegetable & Leaves & 16.67 \\
\hline
\end{tabular}


Table 3 Edible plant species collected in two arid (Boukoumbé) and semi-arid (Bassila) areas of Benin (Continued)

\begin{tabular}{|c|c|c|c|c|c|c|c|c|c|}
\hline AS098 & Djagou-djagou (N) & Justicia insularis T.Anderson & Acanthaceae & Herb & SG & UD & Vegetable & Leaves & 16.67 \\
\hline AS080 & Koupanouwôkou (D), Akohoun (N) & Ocimum basilicum L. & Lamiaceae & Herb & SG, S & W & $\begin{array}{l}\text { Vegetable, } \\
\text { Spice }\end{array}$ & Leaves & 16.67 \\
\hline AS099 & Mukpétida (D) & Phoenix reclinata Jacq. & Arecaceae & Tree & SG & W & Fruit & Fruits & 16.67 \\
\hline AS100 & Ibo gidi (N) & Saba comorensis (Bojer) Pichon & Apocynaceae & Liana & SG & W & Fruit & Pulp & 16.67 \\
\hline AS101 & Ibo gidi (N) & Saba senegalensis (A.DC.) Pichon & Apocynaceae & Liana & SG & W & Fruit, Beverage & Pulp & 16.67 \\
\hline AS081 & $\begin{array}{l}\text { Mucotamu (D), Isenren, Sansan boto, } \\
\text { Adere (N) }\end{array}$ & Syzygium guineense (Willd.) DC. & Myrtaceae & Shrub & SG, S & W & Fruit & Pulp & 16.67 \\
\hline AS011 & Dipugedi (D) & Trichilia emetica Vahl & Meliaceae & Tree & s & W & Fruit & Aril & 16.67 \\
\hline AS102 & Ekunhun ahun (N) & Ananas comosus (L.) Merr. & Bromeliaceae & Herb & SG & C & Fruit & Fruits & 8.33 \\
\hline AS103 & Agni (N) & $\begin{array}{l}\text { Anogeissus leiocarpa (DC.) Guill. \& } \\
\text { Perr. }\end{array}$ & Combretaceae & Tree & SG & W & Vegetable & $\begin{array}{l}\text { Young } \\
\text { leaves }\end{array}$ & 8.33 \\
\hline AS104 & Gbogboloki (N) & Basella alba L. & Basellaceae & Herb & SG & UD & Vegetable & $\begin{array}{l}\text { Leaves, } \\
\text { Shoot }\end{array}$ & 8.33 \\
\hline AS105 & Asha (N) & Bridelia ferruginea Benth. & Euphorbiaceae & Shrub & SG & W & $\begin{array}{l}\text { Food } \\
\text { processing }\end{array}$ & Bark & 8.33 \\
\hline AS012 & Kunaakoobu (D) & Calotropis procera (Aiton) R.Br. & Asclepiadaceae & Shrub & S & UD & $\begin{array}{l}\text { Food } \\
\text { processing }\end{array}$ & Leaves & 8.33 \\
\hline AS106 & Adjèmanwofô (N) & Celosia trigyna $\mathrm{L}$. & Amaranthaceae & Herb & SG & W & Vegetable & Leaves & 8.33 \\
\hline AS107 & Natataka (L) & Chrysanthellum indicum DC. & Asteraceae & Herb & SG & W & Vegetable & Leaves & 8.33 \\
\hline AS082 & $\begin{array}{l}\text { Demmuda dadaara (D), Osan orombo, } \\
\text { Osan wewe (N) }\end{array}$ & Citrus limon (L.) Burm.f. & Rutaceae & Tree & SG, S & C & Fruit & Fruits & 8.33 \\
\hline AS108 & Omronlugboko (N) & Cochlospermum planchonii Hook.f. & Cochlospermaceae & Shrub & SG & W & Condiment & Rootstock & 8.33 \\
\hline AS109 & Gbolo (N) & $\begin{array}{l}\text { Crassocephalum rubens (Juss. ex } \\
\text { Jacq.) S.Moore }\end{array}$ & Asteraceae & Herb & SG & UD & Vegetable & Leaves & 8.33 \\
\hline AS110 & Kanmblê (L) & Cucurbita moschata Duchesne & Cucurbitaceae & Herb & SG & C & Vegetable & Fruits & 8.33 \\
\hline AS013 & Timammuti (D) & Cymbopogon giganteus Chiov. & Poaceae & Herb & S & W & Spice & Leaves & 8.33 \\
\hline AS111 & Tiyankwoun'ti (D), Gnainrissé angbaman (L) & $\begin{array}{l}\text { Cyphostemma adenocaule (Steud. ex } \\
\text { A.Rich) Wild \& R.B.Drumm. }\end{array}$ & Vitaceae & Herb & SG & W & Vegetable & Leaves & 8.33 \\
\hline AS112 & Odundun odo (N) & Emilia sonchifolia (L.) DC. ex Wight & Asteraceae & Herb & SG & W & Vegetable & Leaves & 8.33 \\
\hline AS014 & Mussantipê (D) & Grewia lasiodiscus K.Schum. & Tiliaceae & Shrub & S & W & Condiment & Fruits & 8.33 \\
\hline AS113 & Anikan gbiju (N) & Hoslundia opposita Vahl & Lamiaceae & Shrub & SG & W & $\begin{array}{l}\text { Vegetable, } \\
\text { Spice }\end{array}$ & Leaves & 8.33 \\
\hline AS114 & Ejinrin (N) & Momordica charantia L. & Cucurbitaceae & Herb & SG & W & Vegetable & Leaves & 8.33 \\
\hline AS115 & Adjan'gulu (N) & Senna occidentalis (L.) Link & $\begin{array}{l}\text { Leg- } \\
\text { Caesalpinioideae }\end{array}$ & Herb & SG & W & Vegetable & Leaves & 8.33 \\
\hline
\end{tabular}


Table 4 Wild species richness and percentages (in brackets) of use types per life form

\begin{tabular}{lrccc}
\hline Species counts & & \multicolumn{3}{c}{ Uses } \\
\cline { 3 - 5 } & & Vegetables & Fruits & Other uses \\
\hline Life forms & & & & \\
Herbaceous & 23 & $22(96 \%)$ & $0(0 \%)$ & $2(9 \%)$ \\
Liana & 4 & $1(25 \%)$ & $3(75 \%)$ & $2(50 \%)$ \\
Shrub & 18 & $7(39 \%)$ & $10(56 \%)$ & $9(50 \%)$ \\
Tree & 25 & $9(36 \%)$ & $20(80 \%)$ & $7(28 \%)$ \\
Counts by use type & 70 & $39(56 \%)$ & $33(47 \%)$ & $20(29 \%)$ \\
\hline
\end{tabular}

Although about $83 \%$ of respondents indicated that they had livestock, livestock breeding differed significantly between the two areas. More farmers were involved in livestock breeding in arid area than in semiarid one (Figure $5, \mathrm{p}<0.001$ ). The majority of them explained that livestock resources are used for household needs (e.g., consumption, cultural ceremonies) and commercialized to earn additional financial resources. They argued that this supplementary resource is crucial not only during food shortage period but also during agricultural work period. Animal species raised were dominated by poultry and small ruminants (Figure 5 ).

Although farmers in Bassila exploit an overall higher edible plant diversity compared to their counterpart in Boukoumbé, this is not necessary translated into resourceconservation practices. Land clearing and land preparation practices developed were region-dependent (Figure 6, $\mathrm{p}<0.001)$. Farmers in the semi-arid area used fire to clear new land. On old fields seedling and sapling are pruned and burnt. They also used human traction and to some extent tractors. We noticed no tillage. In the drier area however, the majority of farmers indicated that they use herbicide to clear land. Animal traction is used for tillage and mulches are systematically incorporated during the tillage as soils are degraded. Soil fertility management practices were also region-dependent (Figure 7, $\mathrm{p}<0.001$ ). Chemical fertilizer, animal manure, mulch incorporation and mulching were more used by farmers in the arid area while farmers in the semi-arid area still relied on slashand-burn cultivation, crop rotation, burning of crop residue and mulches, and mixed-cropping. Similarly, field management practices after harvest were related to region where farmers were living (Figure $8, \mathrm{p}<0.001$ ). Farmers in the semi-arid area left fields for fallow and/or burnt crop residues. They explained that burning crop residues after harvest reduces weed pressure at beginning of the next cropping season. It also avoids attracting the nomadic Fulani people and their herds into their fields because cattle increase soil degradation and make tillage labour difficult at the next cropping season. However, farmers in the arid area left fields for fallow and/or grazed their own cattle and small ruminants on the fields. They explained that crop residues serve as fodder for their livestock and the manure serves as fertilizer to crops. Contrary to others farming practices, there is no significant difference between the two municipalities regarding pest management practices (Figure 9, p = 0.1974). Farmers widely used chemical insecticide in both regions, mainly on cotton and to some extent on cowpea.

\section{Relationship among communities in term of choice of edible plants}

To understand the impact of agroecological context and socio-cultural attributes on edible plants selection by a community, we performed a cluster analysis using villages as operational units (Figures 10, 11 and 12). Each village is dominated by only one sociolinguistic group. The

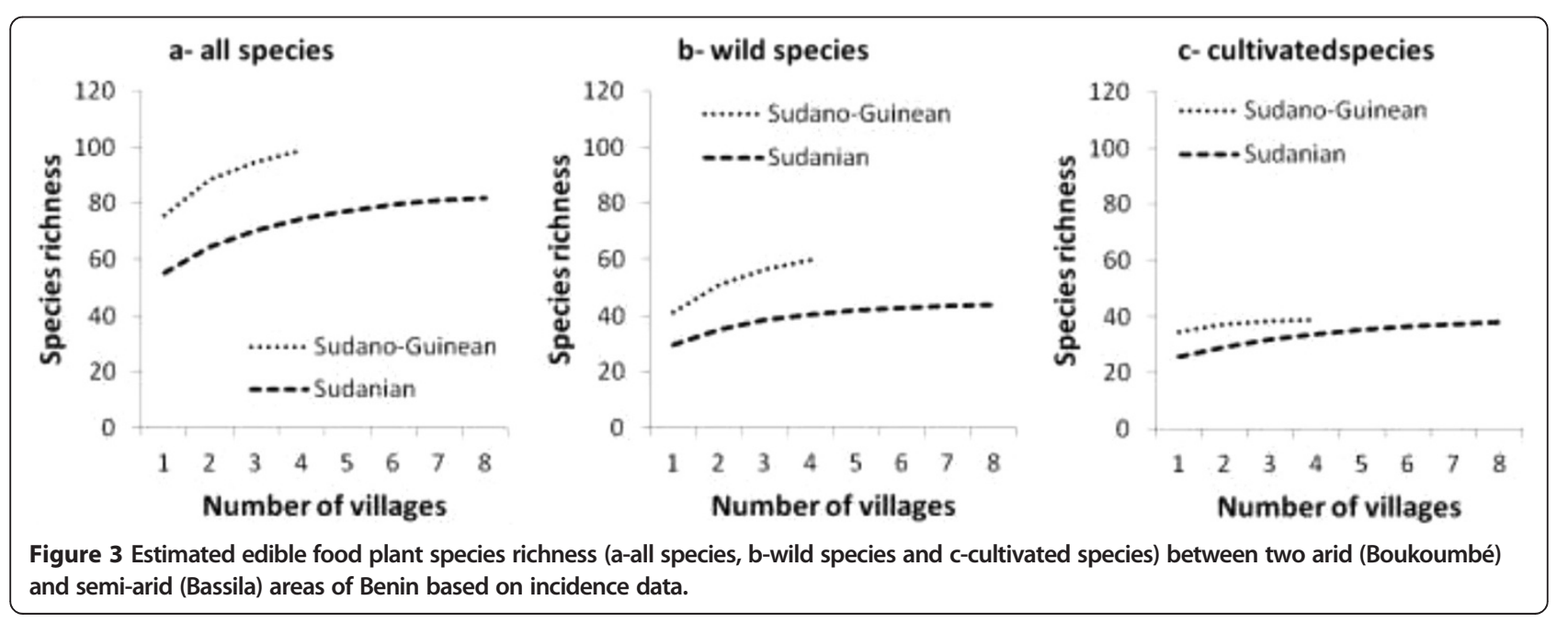



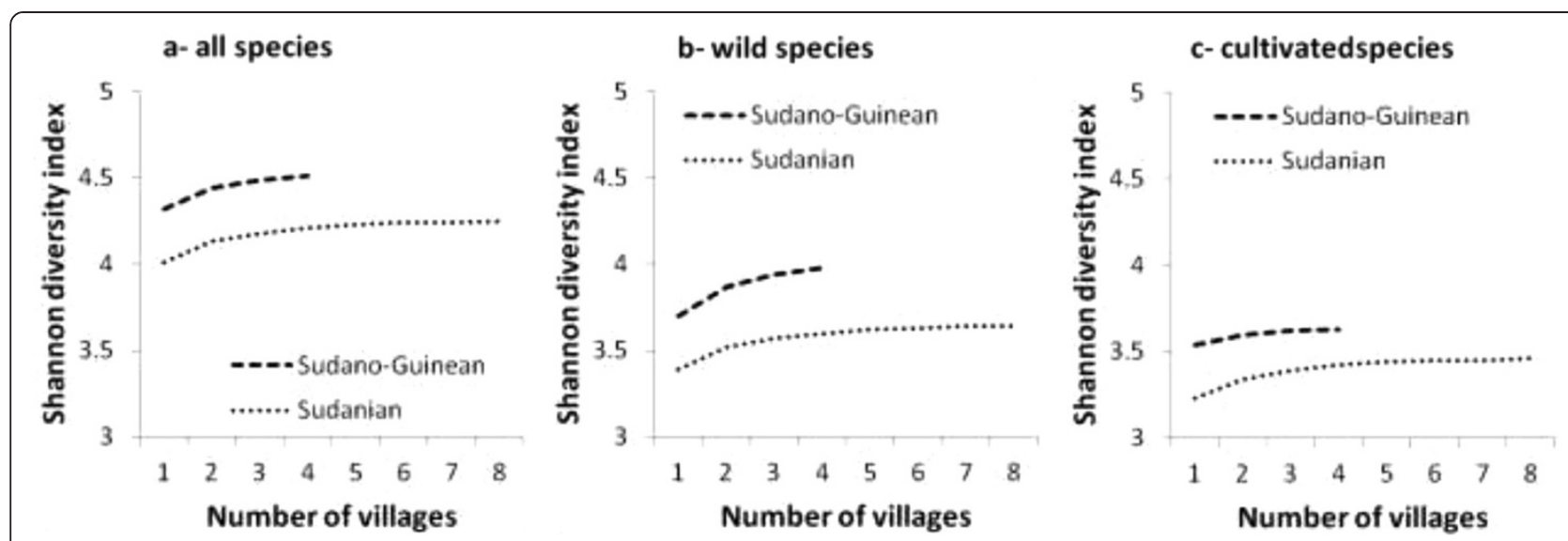

Figure 4 Estimated Shannon diversity index for edible food plant species (a-all species, b-wild species and c-cultivated species) between two arid (Boukoumbé) and semi-arid (Bassila) areas of Benin based on incidence data.

dendrogram in Figure 10 presents data on all edible plants together. At $40 \%$ of similarity, it shows two groups that revealed the two phytogeographical zones. Cluster A1 is composed of all villages of Bassila and no clear grouping according to sociolinguistic membership was noted within the cluster. Cluster A2 is composed of all villages of Boukoumbé. There is no clear grouping according to sociolinguistic membership too. The dendrogram in Figure 11 is based solely on cultivated species, and shows at $40 \%$ of similarity two groups based on phytogeographical zones. Cluster B1 is composed of all villages of Sudano-Guinean municipality and Cluster B2 of all villages of Sudanian municipality. There is no clustering according to sociolinguistic membership. The data in Figure 12 take into account wild species only, the same trend was observed although this topology revealed three clusters: Cluster $\mathrm{C} 1$ composed of all villages of Sudanian region, Cluster C2 composed of one village (Modogui, a Nagot socio-linguistic community) of Sudano-Guinean region and Cluster C3 of the rest of villages (which are of immigrant community) of SudanoGuinean region.

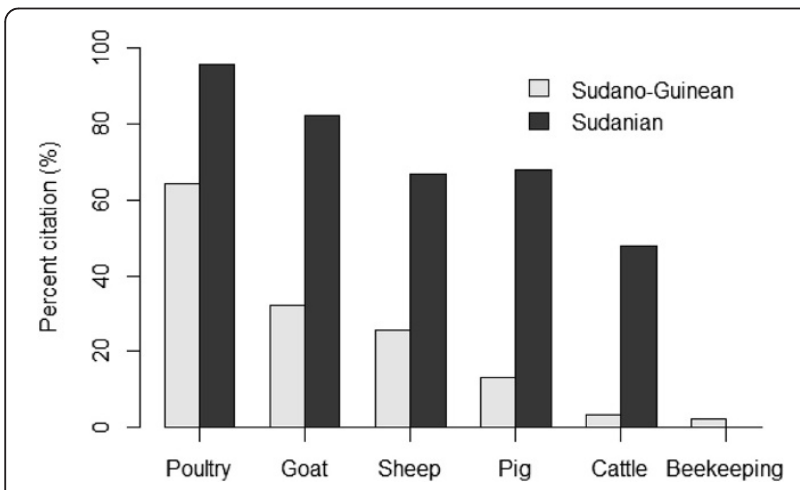

Figure 5 Animal species raised in two arid (Boukoumbé) and semi-arid (Bassila) areas of Benin.

\section{Discussion}

To the best of our knowledge, this is the first empirical study in Benin as well as in West Africa comparing the diversity of edible plants used by communities in two contrasting areas (in terms of ecological and socioeconomic characteristics). Similarly, exploring how communities produce their crops (farming practices' analysis) combined with what they used as food plants (edible food plants diversity analysis) give new insights on local people food basket.

The total number of 115 species consumed as food plants in the two municipalities is considerable. It represents about $4 \%$ of the floristic diversity of the whole country [33]. Among the bulk of food plants used, wild species represent $61 \%$ (70 species) and $46 \%$ of the most cited species by communities. The number of wild edible plants used in the two municipalities represents $43 \%$ of the total number of non-wood edible forest plant resources collected during a country-wide market survey

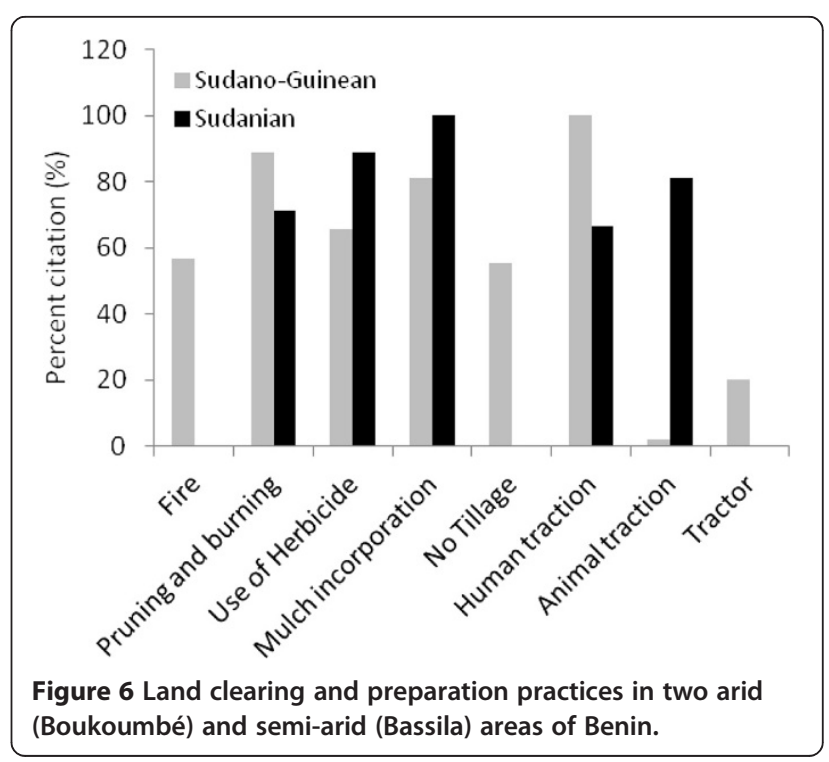




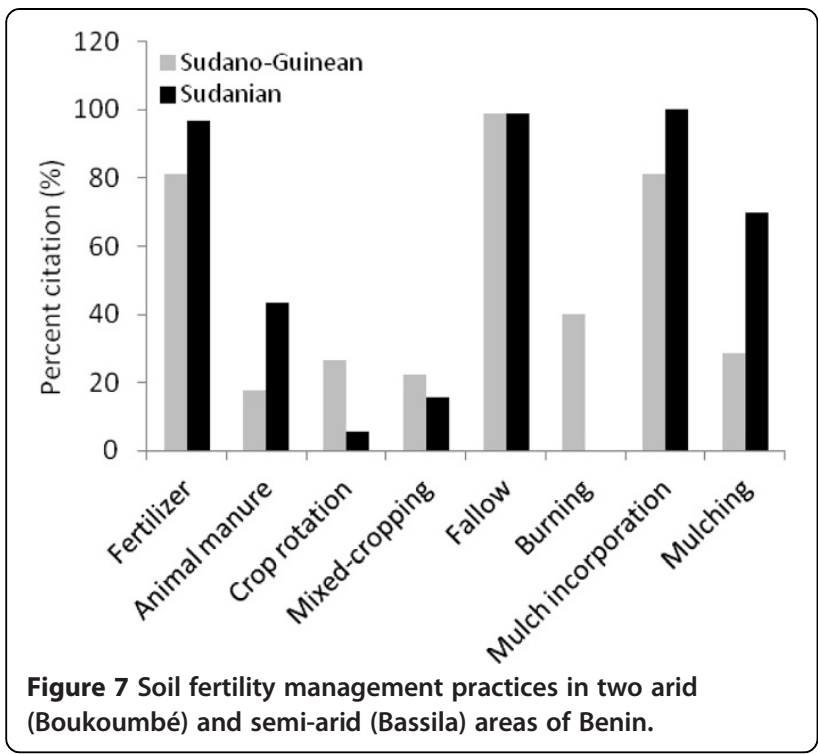

in Benin [52] and is lower than the diversity of noncultivated plant species (87 species) collected in the southwest Benin and the southeast Togo [53]. Vegetable species represent about $57 \%$ while fruit species account for roughly $47 \%$ of wild edible plants collected. This highlights the potential role of wild vegetables and fruits in improving food and nutritional security of rural communities [54-56]. Wild species provide various food products for household's daily diets, especially for poorer households in northern Benin [40]. Our results indicate that in dry areas wild plants still constitute an important asset in addressing food security by ensuring the availability and accessibility of food plants. Their contribution to food security in rainforest regions has also been demonstrated [57,58]. According to Bharucha and Pretty [59] wild plants and animals continue to form a significant proportion of the global food basket, and their roles and values in agricultural systems may be set to grow as

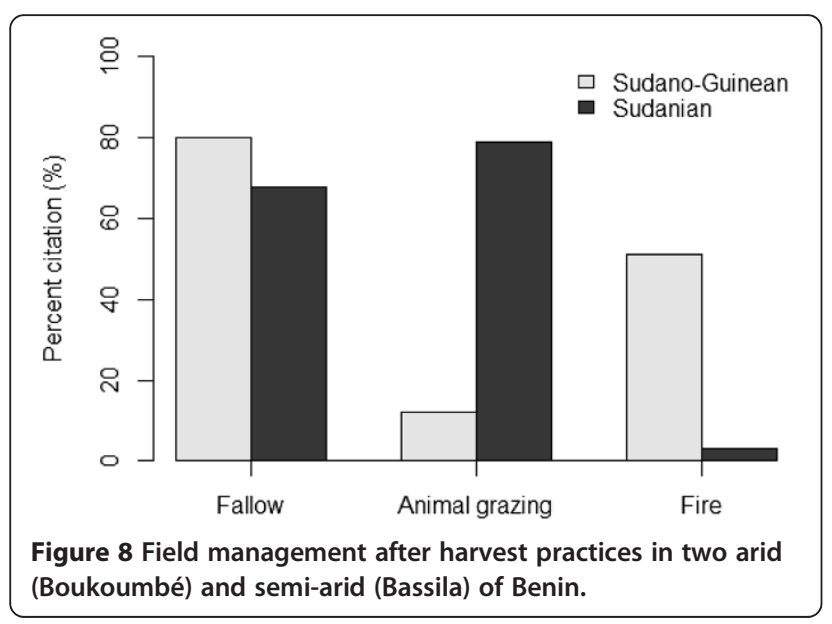

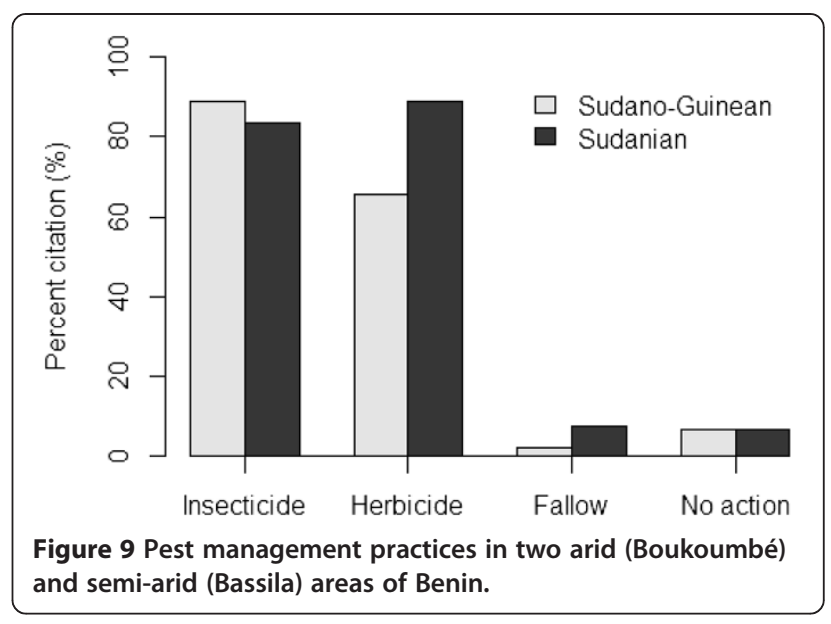

pressures on agricultural productivity increase. Based on our results, we emphasize the important value of wild edible resources in food consumption in dry areas. Their roles in farmers' global livelihoods are likely to be more important, as reported by many authors [22,29,40,57,60-62]. The improvement of food security in developing countries will depend on the placement of wild edible resources in agricultural policies as well. A thorough documentation of wild edible plants and their contribution to household diet will help improve knowledge on the under-valued biological and cultural diversity that are of importance to address food security, environmental and economic sustainability [63]. Moreover, a diversification with greater use of highly valuable but presently under-utilized crops and species should be an essential element of any model for sustainable smallholder agriculture [4].

About $25 \%$ of wild edible plants are under domestication and can be found in one or other step in the "bringing

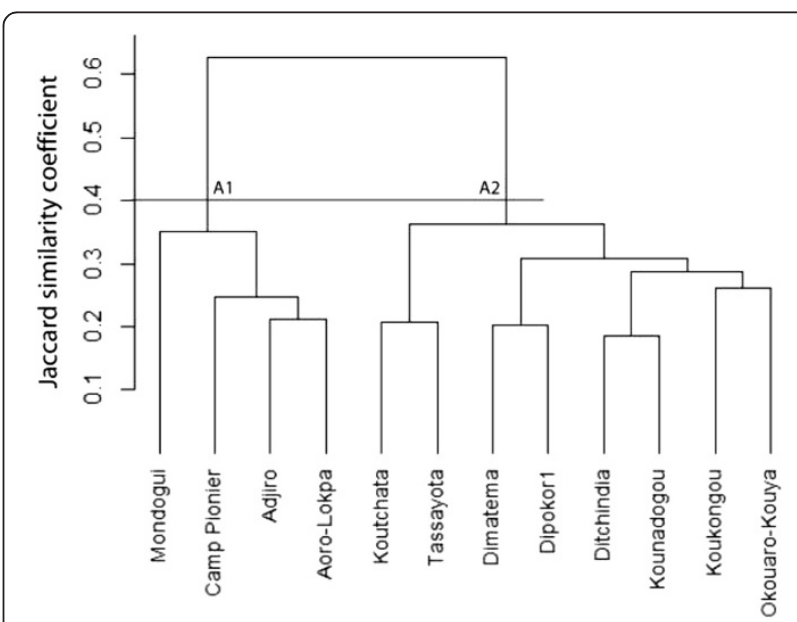

Figure 10 Dendrogram showing the similarity among sociolinguistic groups based on all edible plant species in two arid (Boukoumbé) and semi-arid (Bassila) areas of Benin. 


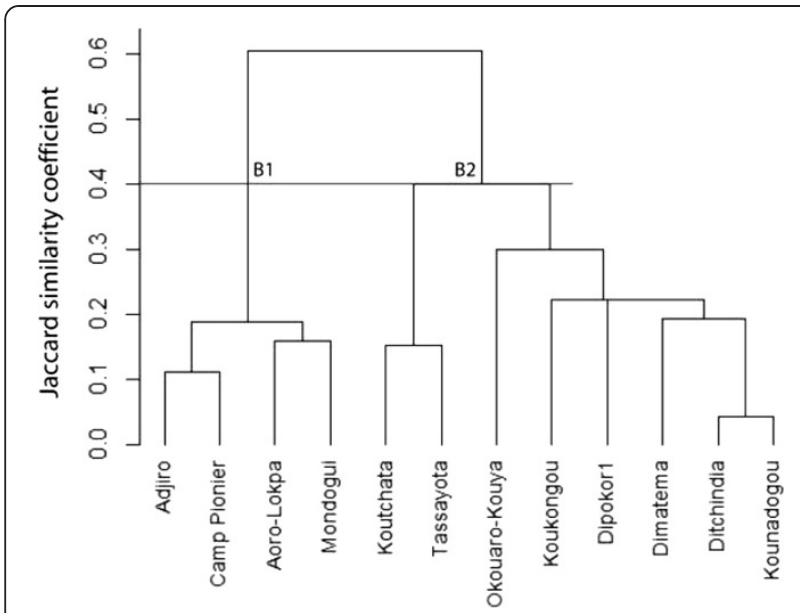

Figure 11 Dendrogram showing the similarity among sociolinguistic groups based on cultivated species in two arid (Boukoumbé) and semi-arid (Bassila) areas of Benin.

into cultivation" phase of the plant domestication process described by Vodouhè and Dansi [64]. Some species are entirely wild and collected only when needed, others are maintained in agricultural environments (fields and fallow) or around habitats and others have dual status depending on the village. Asteraceae and Cucurbitaceae families, which are among the most important plant families, also showed a high vegetable species richness in Benin and Togo $[24,30]$. These findings reveal the importance of these species and botanical families to farmers and to food diets as well. Indeed, food uses is the main reason that motivates local communities in Benin for plant domestication [64]. Therefore, there is a need to consolidate the on-going researches on domestication process of some of species such as $S$. radiatum, J. tenella, A. digitata, $V$. doniana, $C$.

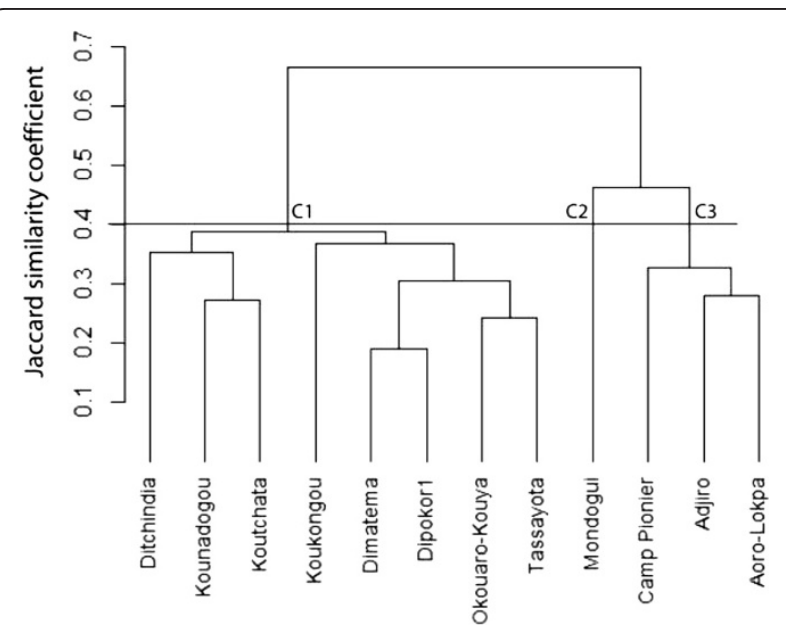

Figure 12 Dendrogram showing the similarity among sociolinguistic groups based on wild species in two arid (Boukoumbé) and semi-arid (Bassila) areas of Benin. rubens and C. crepidioides [24,60,65-71] to ensure and enhance the availability, accessibility, and utilization food plants. Moreover, the promotion of the "bringing into cultivation" practices contributes to not only plant domestication but also to promoting diversity, increasing its sustainable utilization and conservation of agrobiodiversity in situ [64].

Trees, shrubs and lianas account for about $70 \%$ of wild edible plants collected. Many of them are maintained in agricultural environment forming the so called "agroforestry parklands", a widespread traditional land use system in West African dry savannas in which trees and shrubs are intentionally spared and let scattered on farmlands and fallows [72]. Indeed, agroforestry systems offer a number of ecosystem services and environmental benefits, including soil fertility improvement, soil and water conservation, and environmental protection by maintaining ecological stability and conservation of biodiversity [73]. These ecosystem functions have been at the centre of the local ecological knowledge guiding the management options of the farmers [74]. As evidenced by Assogbadjo et al. [75] wild edible species retained in agroforestry systems in Benin depends on farmers' knowledge on species contribution to food, its use in traditional medicine and ceremonies and farmers' perception of its availability in natural vegetation. By regulating ecosystem functions such as nutrient recycling, water use, species diversity and agrochemical pollution agroforestry can sustain agricultural intensification and food security in Africa [76,77]. Moreover, agroforestry systems promote integrated management systems that relate livelihoods and ecosystem service functions to agricultural production and is therefore often considered as a way to sustainably intensify farming practices for enhanced food security, using socially and cost-effective management techniques $[76,78]$. Thus, the improvement of agroforestry practices will benefit wild edible plants and enhance the role of these systems in biodiversity conservation and food provision.

Our study revealed that edible species richness and diversity declines from the semi-arid to the arid zone. Moreover, the diversity of wild edible plants used in Bassila (59 species) is higher than in the buffer zone of the Lama forest in southern Benin (48 botanically identified plants) [58]. It is also higher than in other localities in the Sudano-Guinean region (41 wild edible plants in the buffer zone of the Dan forest in Djidja District [60] and 40 wild edible plants in the Collines region in central Benin [21]). These findings are consistent with results from Achigan-Dako et al. [24] and Salako et al. [79] in Benin on traditional vegetable and home gardens species respectively. This trend could be explained by the fact that in West Africa diversity tends to decline with declining precipitation and from south to north [80]. This southnorth climatic gradient which results from the combining 
effects of annual rainfall, length and severity of the dry season, and air humidity, the soils and geological factors are the major environmental factors underlying plant diversity patterns [81]. Other socio-cultural drivers may also explain this situation. Indeed, the Sudano-Guinean region is a transitional zone between the humid Guinean region in the south and the drier Sudanian region in the north and is a receptacle of strong dynamic migration [23]. The transitional attribute combined with cultural exchange and population migrations within the region could contribute to the observed greater edible species richness and diversity. Cultivated species richness and composition did not significantly differ between the semi-arid and the arid areas. Therefore, the overall difference observed in species richness among the two regions is attributed to wild species. However, as evidenced by Mulumba et al. [82], the use of crop varietal diversity is a risk-minimizing strategy, we speculate that varietal diversity and richness used might be higher in the arid Sudanian zone.

Achieving food security in arid and semi-arid regions of Benin requires productive agriculture, and the wide range of edible plant diversity and farming practices [2] should not be overlooked. A comparative analysis of farming system between the two regions indicated that farming practices were significantly related to phytogeographical regions except for pest management practices. Slash-andburn is still ongoing in semi-arid Sudano-Guinean area as environmental conditions (e.g. precipitation, soil, plant diversity) are more favourable. Fire and burning were heavily used in land preparation, soil fertility management and field management after harvest. New land is converted each year to agriculture, reducing forests and savannas. Cotton expansion is a main driver of forests, savannas and land degradation resulting in loss of biodiversity in West African savannas [83-85]. In the arid Sudanian area farmers have developed more resource-conserving practices as a consequence of land degradation and climatic constraints that they face. They integrate more livestock resources to meet their livelihood needs. As a result they use animal manure for soil fertility management, animal traction for tillage, and graze their livestock on their fields after harvest. We recommended that extension services take into account differences between the two areas and provide farmers with insights and technologies on appropriate crops based on land and resources availability. At the same time, account should be given to intensive awareness-raising about best farming practices. Sustainable or resource-conserving farming practices developed by farmers in less favourable areas need to be strengthened and supported, and also promoted in more favourable areas so as to preserve agroecosystem and natural resources for upcoming generations. Indeed, appropriate agricultural management practices are critical to realizing the benefits of ecosystem services and reducing disservices from agricultural activities [86]. Moreover, the strengthening of livestock-crop integration is crucial for ecological intensification of agriculture to achieve current and future food security and environmental sustainability [87].

Many findings highlighted the importance of sociocultural attributes in the utilization and values that a community gives to plant resources [14-16,18-22]. This is illustrated in Figures 10 and 12, where socio-cultural attributes are indicated to play an important role in plant use. For instance, in Cluster A1 Modogui, a Nagot sociolinguistic group village stands alone; Aoro-Lokpa and Adjiro, two Lokpa villages, are grouped into the same sub-cluster while Camp pionier, a Ditamari sociolinguistic group stands alone. Cluster A2 is composed of Ditamari and M'Bermé, two ethnic groups that have high linguistic and cultural affinities. These two ethnic groups formed with other minor ethnic groups the Otamari linguistic group. Ditamari (in general term Otamari group) and Lokpa from the Sudanian region are two ethnic groups that are the principal actors of migratory dynamics in Benin [23,36]. Members of these groups are motivated to leave their homes in Atacora because of soil degradation, in search of the virgin and fertile lands [23,36,37]. A more clear trend is observed when considering solely wild species, where Nagot ethnic group stands alone in one cluster, immigrant ethnic groups stand alone in Sudano-Guinean zone and Otamari linguistic group stand alone too. However, sociocultural attributes are not sufficient to explain trends revealed by the dendrograms. Villages were mainly clustered according to phytogeographical regions. Camp pionier, a Ditamari village in Sudano-Guinean, does not group with others Ditamari villages in Sudanian region. Bio-geographical factors also play a role in the choice of edible plants by community as also demonstrated for traditional vegetables [24] and for yams' varietal diversity [23] in Benin. We conclude that there is a complementarity between socio-cultural attributes of community and biogeographical factors that may explain the choice of edible food plants, especially for wild species. This conclusion is consistent with previous results that had shown that the knowledge and consumption of wild edible plants follows a pattern according to ecological conditions of the gathering environments, as well as the cultural heritage of the communities $[24,88]$. Our results also indicated that when a community moved to a more favourable area (e.g. precipitation, soil, plant diversity), members adapted their choice to the plant resources available. Other variables such as distance to markets and urbanization may also affect the choice of edible plants. But, here we are in a context of smallholders farming, and agricultural activities are mainly oriented towards households' subsistence. Distance to markets and urbanization were not tested in our study areas which are rural settings. 


\section{Conclusions}

This study revealed the diversity and richness of edible foods plants and analyzed farming practices in arid and semi-arid areas in Benin. We found that wild species play an important role in food consumption of communities in dry areas, and that the diversity of edible plants is higher in the semi-arid area than in the arid one. However, farmers in the less favourable arid area developed advanced resourceconserving practices compared to their counterparts of the favourable semi-arid area. We conclude that if food security has to be addressed, the production and consumption policies must be re-oriented toward the recognition of the place of wild edible plants and farm management practices developed by farmers. For this to happen we suggest a number of policy and strategic decisions as well as research and development actions, including: (1) intensive awareness raising on best farming practices; (2) thorough documentation of wild edible plants and their contribution to household diet; (3) promotion of the "bringing into cultivation" practices; (4) strengthening of livestock-crop integration; and (5) the improvement of agroforestry systems.

There is a room for further investigations on how climatic gradients shape the utilization patterns of crop varietal diversity. This will help develop a sound on-farm conservation approach of plant genetic resources. Moreover, since the frequency of consumption of each species was not documented, more investigations with adapted methodology are therefore required to better understand the importance of each species in the diets. Twenty-Four Hour Recall method (see [58]) is a useful approach that can help gather information regarding diets and nutritional habits.

\section{Competing interests}

The authors declare that they have no competing interests.

\section{Authors' contributions}

ACS and EGAD contributed to the conception and design of the study. ACS collected and analyzed the data, contributed to interpretation of the findings, and prepared the first draft of the manuscript and improved it. EGAD supervised data collection, contributed to interpretation of the findings and improved the manuscript. Both authors read and approved the final manuscript.

\section{Acknowledgements \\ This work was financially supported by New Alliance Trust (Grant No: NAT013/002 to the first author). We gratefully acknowledge the valuable technical and logistic assistance of Damien Coukpo during the fieldwork in Bassila. We are particularly indebted to Géraud NTia and his family for their support, linguistic assistance and collaboration in Boukoumbé. We are also grateful to all the farmers interviewed during this work for their time and willingness to share their knowledge.}

Received: 9 July 2014 Accepted: 9 December 2014

Published: 23 December 2014

\section{References}

1. Jackson LE, Brussaard L, de Ruiter PC, Pascual U, Perrings C, Bawa K: Agrobiodiversity. In Encyclopedia of Biodiversity (Second Edition). Edited by Levin SA. Waltham: Academic; 2013:126-135.
2. Lenné J, Wood D: Agrobiodiversity Management for Food Security: A Critical Review. Wallingford, UK: CAB International; 2011.

3. Thrupp LA: The Importance of Biodiversity in Agroecosystems. J Crop Improv 2004, 12:315-337.

4. Kahane R, Hodgkin T, Jaenicke H, Hoogendoorn C, Hermann M, Keatinge JDH, d'Arros Hughes J, Padulosi S, Looney N: Agrobiodiversity for food security, health and income. Agron Sustain Dev 2013, 33:671-693.

5. Kremen C, lles A, Bacon C: Diversified Farming Systems: An Agroecological, Systems-based Alternative to Modern Industrial Agriculture. Eco/ Soc 2012, 17:44.

6. Love B, Spaner D: Agrobiodiversity: Its Value, Measurement, and Conservation in the Context of Sustainable Agriculture. J Sustain Agric 2007, 31:53-82.

7. Koohafkan P, Altieri MA, Gimenez EH: Green Agriculture: foundations for biodiverse, resilient and productive agricultural systems. Int J Agric Sustain 2011, 10:61-75.

8. Tscharntke T, Clough Y, Wanger TC, Jackson L, Motzke I, Perfecto I, Vandermeer J, Whitbread A: Global food security, biodiversity conservation and the future of agricultural intensification. Biol Conserv 2012, 151:53-59.

9. van Ginkel M, Sayer J, Sinclair F, Aw-Hassan A, Bossio D, Craufurd P, Mourid M, Haddad N, Hoisington D, Johnson N, Velarde CL, Mares V, Mude A, Nefzaoui A, Noble A, Rao KPC, Serraj R, Tarawali S, Vodouhe R, Ortiz R: An integrated agro-ecosystem and livelihood systems approach for the poor and vulnerable in dry areas. Food Secur 2013, 5:751-767.

10. Segnon AC: Ecological intensification options and the place of plant genetic resources in arid and semi-arid areas of Benin. In Master Thesis. Benin: University of Abomey-Calavi, Faculty of Agronomic Sciences; 2014.

11. Hengsdijk $H$, van Keulen $H$ : The effect of temporal variation on inputs and outputs of future-oriented land use systems in West Africa. Agric Ecosyst Environ 2002, 91:245-259.

12. Wheeler T, von Braun J: Climate change impacts on global food security Science 2013, 341:508-513.

13. Garnett T, Appleby MC, Balmford A, Bateman IJ, Benton TG, Bloomer P, Burlingame B, Dawkins M, Dolan L, Fraser D, Herrero M, Hoffmann I, Smith P, Thornton PK, Toulmin C, Vermeulen SJ, Godfray HC: Sustainable Intensification in agriculture: Premises and policies. Science 2013, 341:33-34.

14. Koura K, Ganglo J, Assogbadjo A, Agbangla C: Ethnic differences in use values and use patterns of Parkia biglobosa in Northern Benin. J Ethnobiol Ethnomed 2011, 7:42.

15. Gouwakinnou G, Lykke A, Assogbadjo A, Sinsin B: Local knowledge, pattern and diversity of use of Sclerocarya birrea. J Ethnobiol Ethnomed 2011, 7:1-9.

16. Ekué MRM, Sinsin B, Eyog-Matig O, Finkeldey R: Uses, traditional management, perception of variation and preferences in ackee (Blighia sapida K.D. Koenig) fruit traits in Benin: implications for domestication and conservation. J Ethnobiol Ethnomed 2010, 6:1-14.

17. Ayantunde AA, Briejer M, Hiernaux P, Udo HJ, Tabo R: Botanical knowledge and its differentiation by age, gender and ethnicity in Southwestern Niger. Hum Ecol 2008, 36:881-889.

18. Achigan-Dako EG, Fagbemissi $R$, Avohou HT, Vodouhè SR, Coulibaly $O$, Ahanchédé A: Importance and practices of Egusi crops (Citrullus lanatus (Thunb.) Matsum. \& Nakai, Cucumeropsis mannii Naudin and Lagenaria siceraria (Molina) Standl. cv. 'Aklamkpa') in sociolinguistic areas in Benin. Biotechnol Agron Soc Environ 2008, 12:393-403.

19. Heubach K, Wittig R, Nuppenau E-A, Hahn K: Local values, social differentiation and conservation efforts: The impact of ethnic affiliation on the valuation of NTFP-species in Northern Benin, West Africa. Hum Ecol 2013, 41:513-533.

20. Vodouhê F, Coulibaly O, Greene C, Sinsin B: Estimating the local value of Non-Timber Forest Products to Pendjari Biosphere Reserve dwellers in Benin. Econ Bot 2009, 63:397-412.

21. Avohou HT, Vodouhe RS, Dansi A, Bellon M, Kpeki B: Ethnobotanical factors influencing the use and management of wild edible plants of agricultural environments in Benin. Ethnobotany Res App/ 2012, 10:571-592.

22. Fandohan B, Assogbadjo A, Kakaï R, Kyndt T, Caluwé E, Codjia J, Sinsin B: Women's traditional knowledge, use value, and the contribution of tamarind (Tamarindus indica L.) to rural households' cash income in Benin. Econ Bot 2010, 64:248-259.

23. Baco MN, Biaou G, Lescure J-P: Complementarity between geographical and social patterns in the preservation of yam (Dioscorea sp.) diversity in Northern Benin. Econ Bot 2007, 61:385-393. 
24. Achigan-Dako EG, N'Danikou S, Assogba-Komlan F, Ambrose-Oji B, Ahanchede A, Pasquini MW: Diversity, geographical, and consumption patterns of traditional vegetables in sociolinguistic communities in Benin: Implications for domestication and utilization. Econ Bot 2011, 65:129-145.

25. Van der Stege C, Prehsler S, Hartl A, Vogl CR: Tamarind (Tamarindus indica L.) in the traditional West African diet: not just a famine food. Fruits 2011, 66:171-185.

26. De Caluwé E, De Smedt S, Assogbadjo AE, Samson R, Sinsin B, Van Damme P: Ethnic differences in use value and use patterns of baobab (Adansonia digitata L.) in northern Benin. Afr J Ecol 2009, 47:433-440.

27. Sop TK, Oldeland J, Bognounou F, Schmiedel U, Thiombiano A: Ethnobotanical knowledge and valuation of woody plants species: a comparative analysis of three ethnic groups from the sub-Sahel of Burkina Faso. Environ Dev Sustain 2012, 14:627-649.

28. Houehanou TD, Assogbadjo AE, Kakaï RG, Houinato M, Sinsin B: Valuation of local preferred uses and traditional ecological knowledge in relation to three multipurpose tree species in Benin (West Africa). Forest Policy Econ 2011, 13:554-562.

29. Faye MD, Weber JC, Mounkoro B, Dakouo J-M: Contribution of parkland trees to farmers' livelihoods: a case study from Mali. Dev Pract 2010, 20:428-434.

30. Batawila K, Akpavi S, Wala K, Kanda M, Vodouhè R, Akpagana K: Diversité et gestion des légumes de cueillette au Togo. Afr J Food Agric Nutr Dev 2007, 7:21.

31. FAO, IFAD, WFP: The State of Food Insecurity in the World 2013. The multiple dimensions of food security. Rome, Italy: Food and Agriculture Organization of the United Nations (FAO); 2013.

32. Adomou AC: Phytogoegraphy of Benin. In Protection de la nature en Afrique de l'Ouest: une liste rouge pour le Bénin Nature conservation in West Africa: Red List for Benin. Edited by Neuenschwander P, Sinsin B, Goergen G. Ibadan, Nigeria: International Institute of Tropical Agriculture; 2011:14-20

33. Akoègninou A, Van Der Burg W, Van der Maesen L: Flore Analytique du Bénin. Cotonou \& Wageningen: Backhuys Publishers; 2006.

34. INSAE: Troisième Recensement General de la Population et de l'Habitat (RGPH3), Tome 3: caractéristiques socioculturelles et economiques. Cotonou, Bénin: Direction des Etudes Démographiques, Institue National de Statistique Appliquée et d'Economie (INSAE); 2004.

35. Igué AM, Floquet A, Stahr K: Land use and farming systems in Benin. In Adapted Farming in West Africa: issues, potentials and perspectives. Edited by Graef F, Lawrence P, von Oppen M. Stuttgart, Germany: Verlag Ulrich E. Grauer; 2000:219-230.

36. Doevenspeck M: Migrations rurales, accès au foncier et rapports interethniques au sud du Borgou (Bénin): Une approche méthodologique plurielle. Afr Spectr 2004, 39:359-381.

37. Saïdou A, Tossou RC, Kossou D, Sambieni S, Richards P, Kuyper TW: Land tenure and sustainable soil fertility management in central Benin: towards the establishment of a cooperation space among stakeholders. Int J Agric Sustain 2007, 5:195-212.

38. Bongi S, Obama G, Le Dain A-S, Cossi A: République du Bénin: Analyse globale de la vulnérabilité, de la sécurité alimentaire et de la nutrition. Rome, Italie: Programme Alimentaire Mondial (PAM); 2009. http://documents.wfp.org/ stellent/groups/public/documents/ena/wfp203247.pdf.

39. MAEP: Etude préliminaire sur l'état des lieux des interventions en matière de sécurité alimentaire au Bénin. Cotonou, Bénin: Ministère de l'Agriculture, de l'Elevage et de la Pêche; 2013:175.

40. Heubach K, Wittig R, Nuppenau E-A, Hahn K: The economic importance of non-timber forest products (NTFPs) for livelihood maintenance of rural west African communities: A case study from northern Benin. Ecol Econ 2011, 70:1991-2001

41. INSAE: Cahiers des villages et quartiers de ville des Départements de l'Atacora et de la Donga. Cotonou, Bénin: Direction des Etudes Démographiques, Institue National de Statistique Appliquée et d'Economie (INSAE); 2004.

42. Müller JG, Boubacar R, Dan Guimbo I: The "How" and "Why" of Including Gender and Age in Ethnobotanical Research and Community-Based Resource Management. AMBIO 2014, 1-12.

43. International Society of Ethnobiology: ISE Code of Ethics (with 2008 additions). 2006. http://ethnobiology.net/code-of-ethics/code-in-english/.

44. Achigan-Dako EG, Pasquini MW, Assogba-Komlan F, N'Danikou S, Yédomonhan H, Dansi A, Ambrose-Oji B (Eds): Traditional Vegetables in Benin. Cotonou, Benin: Institut National des Recherches Agricoles du Bénin: Imprimeries du CENAP; 2010.

45. Arbonnier M: Arbres, Arbustes et Lianes des Zones Sèches d'Afrique de l'Ouest, ze édition. Quae-MNHN: Paris-Versailles; 2009.
46. Estimate S, Colwell RK: Statistical estimation of species richness and shared species from samples. Version 9.1.0. User's Guide and application. 2013 http://purl.oclc.org/estimates.

47. Gotelli NJ, Colwell RK: Estimating species richness. In Biological Diversity: Frontiers in Measurement and Assessment. Edited by Magurran AE, McGill BJ. New York: Oxford University Press; 2010:39-54.

48. Millot G: Comprendre et Réaliser les Tests Statistiques à l'Aide de R: Manuel de Biostatistique. 2èth edition. De Boeck: Bruxelles; 2011.

49. Crawley MJ: The R Book. 2nd edition. Chichester: John Wiley \& Sons; 2013.

50. Borcard D, Gillet F, Legendre P: Numerical Ecology with R. New York: Springer; 2011

51. Core Team R: R: A language and environment for statistical computing. Vienna, Austria: R Foundation for Statistical Computing; 2013. http://www.R-project.org/.

52. Codjia JTC, Assogbadjo AE, Ekué MRM: Diversité et valorisation au niveau local des ressources végétales forestières alimentaires du Bénin. Cah Agric 2003, 12:321-331.

53. Rodenburg J, Both J, Heitkönig IMA, Van Koppen CSA, Sinsin B, Van Mele P, Kiepe P: Land Use and Biodiversity in Unprotected Landscapes: The Case of Noncultivated Plant Use and Management by Rural Communities in Benin and Togo. Soc Nat Resour 2012, 25:1221-1240.

54. Fentahun MT, Hager H: Exploiting locally available resources for food and nutritional security enhancement: wild fruits diversity, potential and state of exploitation in the Amhara region of Ethiopia. Food Secur 2009, 1:207-219.

55. Mavengahama S, McLachlan M, Clercq W: The role of wild vegetable species in household food security in maize based subsistence cropping systems. Food Secur 2013, 5:227-233.

56. Keatinge JDH, Yang RY, Hughes JDA, Easdown WJ, Holmer R: The importance of vegetables in ensuring both food and nutritional security in attainment of the Millennium Development Goals. Food Secur 2011, 3:491-501.

57. Chukwuone NA, Okeke CA: Can non-wood forest products be used in promoting household food security?: Evidence from savannah and rain forest regions of Southern Nigeria. Forest Policy Econ 2012, 25:1-9.

58. Boedecker J, Termote C, Assogbadjo A, Van Damme P, Lachat C: Dietary contribution of Wild Edible Plants to women's diets in the buffer zone around the Lama forest, Benin - an underutilized potential. Food Secur 2014, 6:833-849.

59. Bharucha Z, Pretty J: The roles and values of wild foods in agricultural systems. Philos Trans R Soc Lond B Bio/ Sci 2010, 365:2913-2926.

60. N'Danikou S, Achigan-Dako EG, Wong J: Eliciting local values of wild edible plants in Southern Benin to identify priority species for conservation. Econ Bot 2011, 65:381-395.

61. Schreckenberg K: Products of a managed landscape: non-timber forest products in the parklands of the Bassila region, Benin. Glob Ecol Biogeogr 1999, 8:279-289.

62. Pouliot M: Contribution of "Women's Gold" to West African Livelihoods: The Case of Shea (Vitellaria paradoxa) in Burkina Faso. Econ Bot 2012, 66:237-248

63. Johns T, Powell B, Maundu P, Eyzaguirre PB: Agricultural biodiversity as a link between traditional food systems and contemporary development, social integrity and ecological health. J Sci Food Agric 2013, 93:3433-3442.

64. Vodouhè R, Dansi $A$ : The "Bringing into Cultivation" phase of the plant domestication process and its contributions to in situ conservation of genetic resources in Benin. The Sci World J 2012, 2012:13.

65. Adjatin A, Dansi A, Eze CS, Assogba P, Dossou-Aminon I, Akpagana K Akoègninou A, Sanni A: Ethnobotanical investigation and diversity of Gbolo (Crassocephalum rubens (Juss. ex Jacq.) S. Moore and Crassocephalum crepidioides (Benth.) S. Moore), a traditional leafy vegetable under domestication in Benin. Genet Resour Crop Evol 2012, 59:1867-1881.

66. Ahohuendo BC, Sinébou VCO, Ahoton LE, Etèka AC, Dansi A, Ahanchédé $A$, Hounhouigan JD, Sanni A, de Foucault B: Phénologie et biologie florale de Sesamum radiatum Schumach. \& Thon., un légume feuille traditionnel en voie de domestication au Bénin. Acta Bot Gall 2012, 159:335-344.

67. Dansi A, Etèka CA, Adéoti K, Orkwor GC, Ahohuendo BC, Loko YL, Vodouhè $\mathrm{R}$ : Black benniseed (Sesamum radiatum Schum. et Thonn.) cultivated as leafy vegetable in Benin. Genet Resour Crop Evol 2012, 59:955-964.

68. Etèka AC, Ahohuendo BC, Dansi A, Assogba-Komlan F, Vodouhè R, Ahoton LE, Ahanchédé A, Sanni A, Hounhouigan DJ: Diversity, cultural practices and domestication of Sesamum radiatum Thonn. ex Hornem and Justicia tenella (Nees) T., two neglected and underutilised traditional leafy vegetables consumed in Benin, African. J Agric Res 2011, 6:5891-5904. 
69. Jensen JS, Bayala J, Sanou H, Korbo A, Ræbild A, Kambou S, Tougiani A, Bouda H-N, Larsen A, Parkouda C: A research approach supporting domestication of Baobab (Adansonia digitata L.) in West Africa. New For 2011, 41:317-335.

70. N'Danikou S, Achigan-Dako EG, Tchokponhoué DA, Assogba Komlan F, Gebauer J, Vodouhè RS, Ahanchédé A: Enhancing germination and seedling growth in Vitex doniana Sweet for horticultural prospects and conservation of genetic resources. Fruits 2014, 69:279-291.

71. Achigan-Dako EG, N'Danikou S, Tchokponhoue DA, Assogba-Komlan F, Larwanou M, Vodouhè RS, Ahanchédé A: Sustainable use and conservation of Vitex doniana Sweet: Unlocking the propagation ability using stem cuttings. J Agric Environ Int Dev 2014, 108:43-62.

72. Boffa JM: Agroforestry parklands in sub-Saharan Africa. Rome, Italy: Food and Agriculture Organization of the United Nations (FAO); 1999.

73. Jose S: Agroforestry for ecosystem services and environmental benefits: an overview. Agrofor Syst 2009, 76:1-10.

74. Bayala J, Sanou J, Teklehaimanot Z, Kalinganire A, Ouédraogo SJ: Parklands for buffering climate risk and sustaining agricultural production in the Sahel of West Africa. Curr Opin Environ Sustain 2014, 6:28-34.

75. Assogbadjo AE, Glèlè Kakaï R, Vodouhê FG, Djagoun CAMS, Codjia JTC, Sinsin B: Biodiversity and socioeconomic factors supporting farmers' choice of wild edible trees in the agroforestry systems of Benin (West Africa). Forest Policy Econ 2012, 14:41-49.

76. Carsan S, Stroebel A, Dawson I, Kindt R, Mbow C, Mowo J, Jamnadass R: Can agroforestry option values improve the functioning of drivers of agricultural intensification in Africa? Curr Opin Environ Sustain 2014, 6:35-40.

77. Garrity DP, Akinnifesi FK, Ajayi OC, Weldesemayat SG, Mowo JG, Kalinganire A, Larwanou M, Bayala J: Evergreen Agriculture: a robust approach to sustainable food security in Africa. Food Secur 2010, 2:197-214

78. Mbow C, Van Noordwijk M, Luedeling E, Neufeldt H, Minang PA, Kowero G: Agroforestry solutions to address food security and climate change challenges in Africa. Curr Opin Environ Sustain 2014, 6:61-67.

79. Salako VK, Fandohan B, Kassa B, Assogbadjo AE, Idohou AFR, Gbedomon RC, Chakeredza S, Dulloo ME, Glele Kakaï R: Home gardens: an assessment of their biodiversity and potential contribution to conservation of threatened species and crop wild relatives in Benin. Genet Resour Crop Evol 2014, 61:313-330.

80. Linder HP: Plant diversity and endemism in sub-Saharan tropical Africa. J Biogeogr 2001, 28:169-182.

81. Adomou AC, Sinsin B, Maesen LG: Notulae Florae Beninensis 12 : Phytosociological and Chorological Approaches to Phytogeography: A Meso-Scale Study in Benin. Syst Geogr Plants 2006, 76:155-178.

82. Mulumba JW, Nankya R, Adokorach J, Kiwuka C, Fadda C, De Santis P, Jarvis DI: A risk-minimizing argument for traditional crop varietal diversity use to reduce pest and disease damage in agricultural ecosystems of Uganda. Agric Ecosyst Environ 2012, 157:70-86.

83. Baudron F, Corbeels M, Monicat F, Giller K: Cotton expansion and biodiversity loss in African savannahs, opportunities and challenges for conservation agriculture: a review paper based on two case studies. Biodivers Conserv 2009, 18:2625-2644.

84. Chougourou D, Houssou S, Ouinakonhan CM: Importance of cotton in rotation farming system and forest degradation in the district of Gogounou (Bénin). Ann Sci Agronomiques 2008, 11:165-178.

85. Clerici N, Bodini A, Eva H, Grégoire J-M, Dulieu D, Paolini C: Increased isolation of two Biosphere Reserves and surrounding protected areas (WAP ecological complex, West Africa). J Nat Conserv 2007, 15:26-40.

86. Power AG: Ecosystem services and agriculture: tradeoffs and synergies. Philos Trans R Soc Lond B Biol Sci 2010, 365:2959-2971.

87. Lemaire G, Franzluebbers A, Carvalho PCDF, Dedieu B: Integrated croplivestock systems: Strategies to achieve synergy between agricultural production and environmental quality. Agric Ecosyst Environ 2014, 190:4-8.

88. Ladio AH, Lozada M: Patterns of use and knowledge of wild edible plants in distinct ecological environments: a case study of a Mapuche community from northwestern Patagonia. Biodivers Conserv 2004, 13:1153-1173.

doi:10.1186/1746-4269-10-80

Cite this article as: Segnon and Achigan-Dako: Comparative analysis of diversity and utilization of edible plants in arid and semi-arid areas in Benin. Journal of Ethnobiology and Ethnomedicine 2014 10:80.

\section{Submit your next manuscript to BioMed Central and take full advantage of:}

- Convenient online submission

- Thorough peer review

- No space constraints or color figure charges

- Immediate publication on acceptance

- Inclusion in PubMed, CAS, Scopus and Google Scholar

- Research which is freely available for redistribution 